\title{
A Systematic Review of Loneliness and Common Chronic Physical Conditions in Adults
}

\author{
Trisha Petitte $^{1}$, Jennifer Mallow ${ }^{1,4}$, Emily Barnes ${ }^{1}$, Ashley Petrone ${ }^{2,3}$, Taura Barr ${ }^{1,2,3,4}$ and Laurie \\ Theeke $e^{1,4}$, \\ ${ }^{1}$ West Virginia University School of Nursing, United State of America \\ ${ }^{2}$ Department of Neurobiology and Anatomy, Center for Neuroscience, West Virginia, United State of America \\ ${ }^{3}$ Center for Basic and Translational Stroke Research, West Virginia, United State of America \\ ${ }^{4}$ Robert Wood Johnson Nurse Faculty Scholar, United State of America
}

\begin{abstract}
Loneliness is a prevalent and global problem for adult populations and has been linked to multiple chronic conditions in quantitative studies. This paper presents a systematic review of quantitative studies that examined the links between loneliness and common chronic conditions including: heart disease, hypertension, stroke, lung disease, and metabolic disorders. A comprehensive literature search process guided by the PRISMA statement led to the inclusion of 33 articles that measure loneliness in chronic illness populations. Loneliness is a significant biopsychosocial stressor that is prevalent in adults with heart disease, hypertension, stroke, and lung disease. The relationships among loneliness, obesity, and metabolic disorders are understudied but current research indicates that loneliness is associated with obesity and with psychological stress in obese persons. Limited interventions have demonstrated long-term effectiveness for reducing loneliness in adults with these same chronic conditions. Future longitudinal randomized trials that enhance knowledge of how diminishing loneliness can lead to improved health outcomes in persons with common chronic conditions would continue to build evidence to support the translation of findings to recommendations for clinical care.
\end{abstract}

Keywords: Chronic Condition, Loneliness, Systematic Review.

\section{INTRODUCTION AND BACKGROUND}

Prevalence rates of loneliness in adult populations ranges from $17 \%$ in U.S. Adults [1] to over $30 \%$ in older adults in Australia [2]. Loneliness has been defined as a unique psychological construct consisting of distressing emotions that are derived from the absence or perceived absence of expected meaningful interpersonal relationships [3]. In addition to being a major predictor of psychological problems like depression [4] and anxiety [5], loneliness is linked to overall morbidity and mortality in adult populations $[6,7]$. More recent literature suggests that loneliness is linked to multiple chronic physical health conditions like cardiovascular diseases [8] such as atherosclerosis [9] and hypertension [8], through inflammatory and neuroendocrine pathways. The purpose of this paper is to present a systematic review of quantitative studies that demonstrate the links between loneliness and common chronic conditions including heart disease, hypertension, stroke, lung disease, obesity, metabolic syndrome, and adult onset diabetes.

\section{METHODS}

The PRISMA method [10] guided the development of this systematic review of loneliness and common chronic

*Address correspondence to this author at the West Virginia University School of Nursing, USA; E-mail: 1theeke@hsc.wvu.edu conditions. A comprehensive literature search was conducted using EBSCOHost with the following databases selected: Academic Search complete, CINAHL with Full Text, ERIC, MEDLINE, PsycARTICLES, and PsycINFO. Loneliness and the name of each common chronic illness were used as keywords for each chronic illness. For hypertension, the search terms of hypertension and high blood pressure were used. The terms of heart disease, stroke, and obesity were used exclusively for these three chronic conditions. For pulmonary conditions the search terms used were 'pulmonary disease' and 'chronic obstructive pulmonary disease'. Diabetes was searched using 'type 2 diabetes' and 'metabolic syndrome'. Limits were set to include only peerreviewed quantitative studies of adults written in the English language. In addition, the Cochrane Library was searched for a review on loneliness and chronic conditions but none were retrieved.

The search netted 171 articles, 21 duplicates were eliminated within the individual chronic conditions (Fig. 1). A total of 150 articles were abstracted and 28 articles were excluded because they were not quantitative study reports but rather posters, narratives, or opinion pieces. Of the 122 articles abstracted for eligibility, 89 were excluded for the following reasons: no measure of loneliness or chronic disease, qualitative research design, caregiver or child samples, literature reviews or sensitivity analysis. Thirty- 


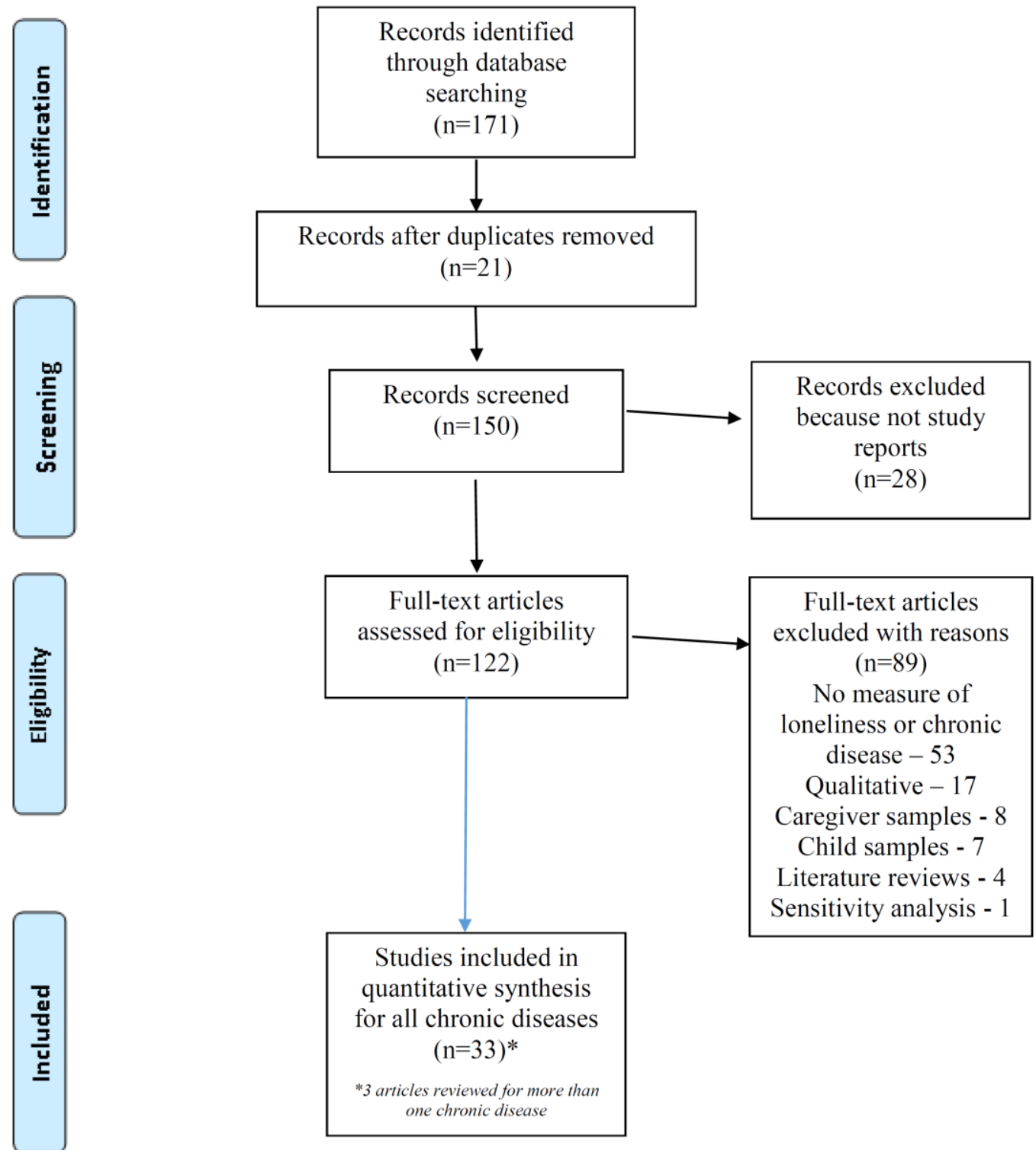

Fig. (1). Literature search result:identification, exclusion, eligibility and included.

three articles were included for all chronic conditions and 3 of the articles were reviewed in the context of more than one chronic conditions. Table 1 presents a summary of each article including the authors, year of publication, location of study, purpose of the study, study design, sample size and target population, data collection methods including study instruments, chronic disease focus, strengths, and weaknesses.

\section{LONELINESS AND HEART DISEASE}

There were a total of 112 articles published between the years of 2000 and 2014 that were considered for inclusion from the literature search for studies of loneliness and heartrelated problems. After removing 16 duplicates and excluding 79 articles for not meeting the inclusion criteria, 16 articles remained. Articles were excluded as follows: 46 did not either measure or report data on loneliness and heart problems, 11 were narratives or stories, 10 were qualitative in design, 6 included child samples, 5 were opinion pieces or columns, 1 was a case study, and 1 was of adults with congenital heart anomalies.

The relationship between the stress of loneliness and heart problems has been described since the 1970's. The sentinel piece of literature, a book entitled "The Broken Heart: The medical consequences of loneliness" [11] described relationships between loneliness, social desirability, importance of love and relationships, health behaviors, morbidity, and mortality in adults in the United States. Since that time, quantitative studies of loneliness and heart disease have focused on varied populations with cardiovascular illness including those with hypertension, coronary heart disease, and heart failure. It is thought that the mechanism of this connection is in the human response to stress which results in a cascade of hormones that influence cardiovascular tone [12], blood pressure [13], and inflammatory responses [9]. 
Table 1. Literature review matrix: purpose, design, data collection characteristics, results and strengths and weaknesses

\begin{tabular}{|c|c|c|c|c|c|c|c|c|}
\hline $\begin{array}{c}\text { (Authors, Year) } \\
\text { Location }\end{array}$ & Aim & Design & Sample Size & Data Collection & CI & Results & Strengths & Weaknesses \\
\hline $\begin{array}{l}\text { (Brouwers et al., } \\
\text { 2014) } \\
\text { Netherlands }\end{array}$ & $\begin{array}{l}\text { To examine } \\
\text { the relative } \\
\text { importance of } \\
\text { inflammation, } \\
\text { disease } \\
\text { severity, and } \\
\text { personality as } \\
\text { predictors of } \\
\text { depression in } \\
\text { HF patients }\end{array}$ & $\mathrm{L}$ & $\begin{array}{l}\mathrm{N}=268 \\
\text { adults }\end{array}$ & $\begin{array}{l}\text { Data collected at } \\
\text { baseline and at one- } \\
\text { year follow-up. }\end{array}$ & $\mathrm{HD}$ & $\begin{array}{l}\text { At baseline, } \\
\text { loneliness was } \\
\text { significantly } \\
\text { related to } \\
\text { depression. } \\
\text { Loneliness was } \\
\text { independently } \\
\text { associated with } \\
\text { higher depression } \\
\text { levels at one-year } \\
\text { follow-up } \\
(\mathrm{p}<.005)\end{array}$ & $\begin{array}{l}\text { Longitudinal } \\
\text { design } \\
\text { included } \\
\text { biomarkers of } \\
\text { disease } \\
\text { severity and } \\
\text { personality } \\
\text { measures }\end{array}$ & $\begin{array}{l}\text { Sample - } \\
60 \% \text { males } \\
\text { with NYHA } \\
\text { class I HF } \\
\text { diagnosis. } \\
\text { Directionalit } \\
\text { y of } \\
\text { relationship } \\
\text { between } \\
\text { inflammation } \\
\text {, disease } \\
\text { severity \& } \\
\text { personality } \\
\text { could not be } \\
\text { assessed. }\end{array}$ \\
\hline $\begin{array}{l}\text { (Cacioppo et al., } \\
\text { 2000) } \\
\text { USA }\end{array}$ & $\begin{array}{l}\text { To investigate } \\
\text { the autonomic } \\
\text { and } \\
\text { neuroendocrin } \\
\text { e responses to } \\
\text { acute stress in } \\
27 \text { women who } \\
\text { are presently } \\
\text { caring for a } \\
\text { spouse and } 37 \\
\text { noncaregivers } \\
\text { matched for } \\
\text { age and family } \\
\text { income }\end{array}$ & $\begin{array}{l}\mathrm{X} \\
\mathrm{DC}\end{array}$ & $\begin{array}{l}64 \text { carers } \\
\text { and non- } \\
\text { carers, } \\
\text { Convenience } \\
\text { sample }\end{array}$ & $\begin{array}{l}\text { Face to face and } \\
\text { physical measures }\end{array}$ & $\mathrm{HD}$ & $\begin{array}{l}\text { Caregivers were } \\
\text { lonelier. }(\mathrm{p}<.05) \\
\text { and caregivers had } \\
\text { greater activation } \\
\text { of the sympathetic } \\
\text { branch of the } \\
\text { autonomic nervous } \\
\text { system, given the } \\
\text { controls, this is } \\
\text { likely the chronic } \\
\text { stress. }\end{array}$ & $\begin{array}{l}\text { NYUL scale } \\
\text { Reliable } \\
\text { measures } \\
\text { Rigorous } \\
\text { measure of BP, } \\
\text { HR, PEP, } \\
\text { RSA, Resp }\end{array}$ & $\begin{array}{l}\text { Small sample } \\
\text { size may } \\
\text { have limited } \\
\text { analysis on } \\
\text { some of the } \\
\text { variables. }\end{array}$ \\
\hline $\begin{array}{l}\text { (Chen, Fettich, \& } \\
\text { McClosky, 2012) } \\
\text { USA } \\
\text { (Chicago) }\end{array}$ & $\begin{array}{l}\text { Examine } \\
\text { whether } \\
\text { weight-related } \\
\text { stigma } \\
\text { increases (1) } \\
\text { the likelihood } \\
\text { of suicidal } \\
\text { ideation and/or } \\
\text { behavior or (2) } \\
\text { the degree of } \\
\text { loneliness; and }\end{array}$ & $\mathrm{X}$ & $\begin{array}{l}396 \text { severely } \\
\text { obese adults } \\
\text { seeking } \\
\text { bariatric } \\
\text { surgery }\end{array}$ & $\begin{array}{l}\text { Weight-related } \\
\text { stigma - } \\
\text { Stigmatizing } \\
\text { Situations } \\
\text { Inventory (SSI) } \\
\text { Loneliness - UCLA } \\
\text { Loneliness Scale } \\
\text { Suicidality - } \\
\text { Suicidal Behaviors } \\
\text { Questionnaire } \\
\text { (SBQ) }\end{array}$ & $\mathrm{O}$ & $\begin{array}{l}\text { Women: Greater } \\
\text { weight-related } \\
\text { stigma was } \\
\text { significantly } \\
\text { associated with } \\
\text { greater loneliness } \\
(S E=1.03, t= \\
2.01) . \\
\text { Greater loneliness } \\
\text { was significantly } \\
\text { associated with }\end{array}$ & $\begin{array}{l}\text { Separate } \\
\text { analysis of men } \\
\text { and women } \\
\text { due to gender } \\
\text { influence on } \\
\text { suicidality. } \\
\text { Use of valid } \\
\text { and reliable } \\
\text { surveys. }\end{array}$ & $\begin{array}{l}\text { Other } \\
\text { mediators for } \\
\text { suicidality } \\
\text { may exist. } \\
\text { Cross- } \\
\text { sectional } \\
\text { design } \\
\text { limited } \\
\text { attempt to } \\
\text { assess } \\
\text { mediation. }\end{array}$ \\
\hline
\end{tabular}


Table 1. Contd....

\begin{tabular}{|c|c|c|c|c|c|c|c|c|}
\hline $\begin{array}{c}\text { (Authors, Year) } \\
\text { Location }\end{array}$ & Aim & Design & Sample Size & Data Collection & CI & Results & Strengths & Weaknesses \\
\hline & $\begin{array}{l}\text { whether } \\
\text { hypotheses (1) } \\
\text { and (2) are } \\
\text { supported (3) } \\
\text { if loneliness } \\
\text { mediates the } \\
\text { effect of } \\
\text { weight-related } \\
\text { stigma on } \\
\text { suicidal } \\
\text { ideation and/or } \\
\text { behavior. }\end{array}$ & & & & & $\begin{array}{l}\text { greater suicidality } \\
(S E=0.01, t= \\
2.01), \text { controlling } \\
\text { for weight-related } \\
\text { stigma. } \\
\text { Men: Greater } \\
\text { weight-related } \\
\text { stigma was } \\
\text { significantly } \\
\text { associated with } \\
\text { greater loneliness } \\
(S E=2.29, t= \\
2.23) . \\
\text { Greater loneliness } \\
\text { was not associated } \\
\text { with suicidality } \\
(S E=0.01, t= \\
0.57), \text { when } \\
\text { controlled for } \\
\text { weight-related } \\
\text { stigma. }\end{array}$ & & $\begin{array}{l}\text { Convenience } \\
\text { sample. } \\
\text { Self-report } \\
\text { prior to } \\
\text { bariatric } \\
\text { surgery } \\
\text { which may } \\
\text { have } \\
\text { influenced } \\
\text { responses. }\end{array}$ \\
\hline $\begin{array}{l}\text { (Hilari et al., } \\
\text { 2010) } \\
\text { United Kingdom }\end{array}$ & $\begin{array}{l}\text { To determine } \\
\text { the factors that } \\
\text { predict } \\
\text { psychological } \\
\text { distress during } \\
\text { the six months } \\
\text { after stroke } \\
\text { including those } \\
\text { with aphasia }\end{array}$ & $\mathrm{L}$ & $\begin{array}{l}\mathrm{N}=87 \text { at } \\
\text { baseline } \\
\mathrm{N}=71 \text { at } 6- \\
\text { months } \\
\text { Ages } 19 \text { to } \\
91\end{array}$ & $\begin{array}{l}\text { General Health } \\
\text { Questionnaire-12, } \\
\text { NIH Stroke Scale, } \\
\text { Barthel Index, } \\
\text { Frenchay Aphasia } \\
\text { Screening Test, } \\
\text { Frenchay Activities } \\
\text { Index, MOS Social } \\
\text { Support Scale }\end{array}$ & $\mathrm{S}$ & $\begin{array}{l}\text { Variables } \\
\text { associated with } \\
\text { distress 6-months } \\
\text { post stroke were: } \\
\text { loneliness } \\
(\mathrm{r}=-0.50, \mathrm{p},< \\
0.001), \\
\text { psychological } \\
\text { distress }(\mathrm{r}=0.45, \mathrm{p} \\
, 0.001), \text { and low } \\
\text { satisfaction with } \\
\text { social network } \\
(\mathrm{r}=-0.29, \mathrm{p}< \\
0.001)\end{array}$ & $\begin{array}{l}\text { Longitudinal } \\
\text { design, } \\
\text { inclusion of } \\
\text { people with } \\
\text { aphasia \& a } \\
\text { wide range of } \\
\text { variables such } \\
\text { as social } \\
\text { factors in an } \\
\text { effort to } \\
\text { determine } \\
\text { prevalent } \\
\text { predictors of } \\
\text { distress post } \\
\text { stroke. }\end{array}$ & $\begin{array}{l}\text { Cognitive } \\
\text { impairment } \\
\text { may be a } \\
\text { predictor of } \\
\text { distress post } \\
\text { stroke, but } \\
\text { was not } \\
\text { measured or } \\
\text { considered. } \\
\text { Exclusion of } \\
\text { people with } \\
\text { severe } \\
\text { receptive } \\
\text { aphasia. }\end{array}$ \\
\hline $\begin{array}{l}\text { (Jacobs, J. M., } \\
\text { Cohen, A., } \\
\text { Hammerman- } \\
\text { Rozenberg, R., \& } \\
\text { Stessman, J., } \\
\text { 2006) } \\
\text { Israel }\end{array}$ & $\begin{array}{l}\text { To describe the } \\
\text { nature of } \\
\text { global sleep } \\
\text { satisfaction } \\
\text { (GSS) of older } \\
\text { people and the } \\
\text { factors } \\
\text { associated with } \\
\text { it. }\end{array}$ & $\mathrm{L}$ & $\begin{array}{l}\mathrm{N}=290 \\
\text { Subjects } \\
\text { recruited } \\
\text { from the } \\
\text { Jerusalem } \\
\text { Cohort Study } \\
\text { (age } 70 \text { at } \\
\text { baseline and } \\
77 \text { at follow } \\
\text { up) }\end{array}$ & $\begin{array}{l}\text { Interview and exam } \\
\text { in hospital (phase } \\
\text { 1) and at home } \\
\text { (phase 2). } \\
\text { GSS - "Are you } \\
\text { satisfied with your } \\
\text { sleep in the last } \\
\text { month?" } \\
\text { ADLs: } 6 \text { items } \\
\text { taken from the Katz } \\
\text { scale } \\
\text { IADLs: } 5 \text { IADLs } \\
\text { analyzed } \\
\text { Depression - Brief } \\
\text { Symptoms }\end{array}$ & $\mathrm{O}$ & $\begin{array}{l}\text { Risk factors at age } \\
70 \text { for subsequent } \\
\text { poor GSS were } \\
\text { loneliness (OR } 3.8, \\
95 \% \text { CI: } 1.8-8.2 \text { ), } \\
\text { depression, poor } \\
\text { self-rated health, } \\
\text { economic } \\
\text { difficulties, back } \\
\text { pain, obesity (OR } \\
2.3,95 \% \text { CI: 1.0- } \\
5.2 \text { ), and prior poor } \\
\text { GSS. } \\
\text { Loneliness was } \\
\text { significantly } \\
\text { associated with } \\
\text { poor GSS ( } p= \\
.002 \text { ) }\end{array}$ & $\begin{array}{l}\text { Sample } \\
\text { representative } \\
\text { of population. }\end{array}$ & $\begin{array}{l}\text { Did not use } \\
\text { standard } \\
\text { measures } \\
\text { (BMI) to } \\
\text { define } \\
\text { obesity. } \\
\text { Data } \\
\text { collected } 7 \\
\text { years apart. }\end{array}$ \\
\hline
\end{tabular}


Table 1. Contd....

\begin{tabular}{|c|c|c|c|c|c|c|c|c|}
\hline $\begin{array}{c}\text { (Authors, Year) } \\
\text { Location }\end{array}$ & Aim & Design & Sample Size & Data Collection & CI & Results & Strengths & Weaknesses \\
\hline & & & & $\begin{array}{l}\text { Inventory } \\
\text { Obesity - defined } \\
\text { as the upper } \\
\text { quartile of body } \\
\text { mass index within } \\
\text { the cohort. }\end{array}$ & & $\begin{array}{l}\text { Poor GSS was } \\
\text { significantly } \\
\text { associated in 70- } \\
\text { and 77-year-olds } \\
\text { with general } \\
\text { fatigue, poor self- } \\
\text { rated health, } \\
\text { depression, } \\
\text { difficulty with one } \\
\text { or more ADLs, } \\
\text { low physical } \\
\text { activity, obesity ( } p \\
=.02), \text { and joint } \\
\text { pain. }\end{array}$ & & \\
\hline $\begin{array}{l}\text { (Jacobs, } \\
\text { Hammerman- } \\
\text { Rozenberg, } \\
\text { Cohen, \& } \\
\text { Stessman, 2006) } \\
\text { Israel }\end{array}$ & $\begin{array}{l}\text { To achieve a } \\
\text { primary } \\
\text { description of } \\
\text { the nature, } \\
\text { prevalence, } \\
\text { and time } \\
\text { course of } \\
\text { chronic back } \\
\text { pain among the } \\
\text { elderly; } \\
\text { identify } \\
\text { significant } \\
\text { associations } \\
\text { with health- } \\
\text { related } \\
\text { variables over } \\
\text { time; and } \\
\text { identify } \\
\text { predictive } \\
\text { factors for the } \\
\text { development } \\
\text { of chronic } \\
\text { back pain in } \\
\text { this age group. }\end{array}$ & $\mathrm{L}$ & $\begin{array}{l}\mathrm{N}=277 \\
\text { (age } 70 \text { at } \\
\text { baseline and } \\
77 \text { at follow } \\
\text { up) }\end{array}$ & $\begin{array}{l}\text { Loneliness - Asked: } \\
\text { "Do you ever feel } \\
\text { lonely?" with } \\
\text { possible responses } \\
\text { never, very rarely, } \\
\text { occasionally, and } \\
\text { very often. } \\
\text { Loneliness was } \\
\text { defined as any } \\
\text { positive response. } \\
\text { Obesity - defined } \\
\text { as the upper decile } \\
\text { of body mass index } \\
\text { within the cohort. }\end{array}$ & $\mathrm{O}$ & $\begin{array}{l}\text { At both ages ( } 70 \\
\text { and } 77) \text {, chronic } \\
\text { back pain was } \\
\text { more frequent } \\
\text { among those } \\
\text { reporting } \\
\text { loneliness ( } P \\
<0.001) \text {. } \\
\text { Chronic back pain } \\
\text { was associated } \\
\text { with obesity at } \\
\text { both ages }(P=0.06 \\
\text { and } P=0.59, \\
\text { respectively). } \\
\text { Loneliness is an } \\
\text { independent risk } \\
\text { factor at age } 70 \text { for } \\
\text { Chronic back pain } \\
\text { (Regression } \\
\text { coefficient }=1.54 \text {, } \\
\text { OR = 4.60, } 95 \% \text { CI } \\
1.19-17.71) \text {. }\end{array}$ & $\begin{array}{l}\text { Representative } \\
\text { sample. } \\
\text { Longitudinal } \\
\text { design. }\end{array}$ & $\begin{array}{l}\text { Measure of } \\
\text { obesity is not } \\
\text { the } \\
\text { commonly } \\
\text { accepted } \\
\text { definition of } \\
\text { BMI > 30 } \\
\text { (they used } \\
\text { top decile of } \\
\text { sample). } \\
\text { Single } \\
\text { question to } \\
\text { evaluate } \\
\text { loneliness. }\end{array}$ \\
\hline $\begin{array}{l}\text { (Jongenelis et al., } \\
\text { 2004) } \\
\text { Amsterdam, } \\
\text { Netherlands }\end{array}$ & $\begin{array}{l}\text { To classify the } \\
\text { contributions } \\
\text { of different } \\
\text { socioeconomic } \\
\text { and dietary } \\
\text { factors that } \\
\text { contribute to } \\
\text { obesity }\end{array}$ & M & $\begin{array}{l}\mathrm{N}=350 \text { Ages } \\
55 \text { and older }\end{array}$ & $\begin{array}{l}\text { Geriatric } \\
\text { Depression Scale } \\
\text { (GDS), Schedule of } \\
\text { Clinical } \\
\text { Assessment in } \\
\text { Neuropsychiatry, } \\
\text { 11-item Loneliness } \\
\text { Scale, Social } \\
\text { Support - SSL12-I }\end{array}$ & $\mathrm{S}$ & $\begin{array}{l}\text { Loneliness was } \\
\text { associated with } \\
\text { sub-clinical } \\
\text { depression (OR= } \\
3.44,95 \% \text { CI 1.90- } \\
6.21) \text {; minor } \\
\text { depression }(\mathrm{OR}= \\
\text { 4.52, 2.06-9.90); } \\
\text { and major } \\
\text { depression }(\mathrm{OR}= \\
\text { 13.37, 3.08-58.15) }\end{array}$ & $\begin{array}{l}\text { Depression } \\
\text { assess by both } \\
\text { symptom } \\
\text { rating scale } \\
\text { (GDS) and a } \\
\text { diagnostic tool } \\
\text { (DSM IV) } \\
\text { Rigorous } \\
\text { sampling of } \\
\text { nursing home } \\
\text { residents }\end{array}$ & $\begin{array}{l}\text { High drop- } \\
\text { out rate } \\
\text { Cannot } \\
\text { determine } \\
\text { depression } \\
\text { rates prior to } \\
\text { nursing home } \\
\text { admission } \\
\text { therefore } \\
\text { causal } \\
\text { relations } \\
\text { could not be } \\
\text { assessed in } \\
\text { depression \& } \\
\text { risk } \\
\text { indicators }\end{array}$ \\
\hline
\end{tabular}


Table 1. Contd....

\begin{tabular}{|c|c|c|c|c|c|c|c|c|}
\hline $\begin{array}{c}\text { (Authors, Year) } \\
\text { Location }\end{array}$ & Aim & Design & Sample Size & Data Collection & CI & Results & Strengths & Weaknesses \\
\hline $\begin{array}{l}\text { (Kara \& Mirici, } \\
\text { 2004) } \\
\text { Turkey }\end{array}$ & $\begin{array}{l}\text { To explore the } \\
\text { differences in } \\
\text { perceptions of } \\
\text { loneliness, } \\
\text { depression, } \\
\text { and social } \\
\text { support among } \\
\text { people with } \\
\text { COPD and } \\
\text { their spouses }\end{array}$ & X & $\begin{array}{l}\mathrm{N}=30 \text { People } \\
\text { with COPD } \\
\text { and their } \\
\text { spouses }\end{array}$ & $\begin{array}{l}\text { Loneliness - UCLA } \\
\text { loneliness scale } \\
\text { Depression - Beck } \\
\text { Depression } \\
\text { Inventory } \\
\text { Social support - } \\
\text { Perceived social } \\
\text { support from } \\
\text { Friends and Family } \\
\text { scale }\end{array}$ & $\mathrm{P}$ & $\begin{array}{l}\text { Social support may } \\
\text { help to alleviate } \\
\text { feelings of } \\
\text { loneliness in } \\
\text { patients with } \\
\text { COPD and their } \\
\text { spouses }\end{array}$ & $\begin{array}{l}\text { Three reliable } \\
\text { instruments } \\
\text { used to } \\
\text { measure } \\
\text { loneliness and } \\
\text { contributing } \\
\text { factors }\end{array}$ & $\begin{array}{l}\text { Small sample } \\
\text { size, } \\
\text { replication of } \\
\text { previous } \\
\text { research }\end{array}$ \\
\hline $\begin{array}{l}\text { (Keele-Card, } \\
\text { Foxall, \& Barron, } \\
\text { 1993) } \\
\text { USA (Midwest) }\end{array}$ & $\begin{array}{l}\text { To explore the } \\
\text { differences in } \\
\text { perceptions of } \\
\text { loneliness, } \\
\text { depression, } \\
\text { and social } \\
\text { support among } \\
\text { people with } \\
\text { COPD and } \\
\text { their spouses }\end{array}$ & $X$ & $\begin{array}{l}\mathrm{N}=30 \text { People } \\
\text { with COPD } \\
\text { and their } \\
\text { spouses }\end{array}$ & $\begin{array}{l}\text { UCLA loneliness } \\
\text { scale } \\
\text { Center for } \\
\text { Epidemiological } \\
\text { Studies Depression } \\
\text { scale } \\
\text { Social support } \\
\text { questionnaire } \\
\text { (SSQ }_{6} \text { ) }\end{array}$ & $\mathrm{P}$ & $\begin{array}{l}\text { Loneliness exists } \\
\text { among patients } \\
\text { with COPD and } \\
\text { their spouses. } \\
\text { Community home } \\
\text { health nurses need } \\
\text { to be sensitive to } \\
\text { the needs of their } \\
\text { clients and family } \\
\text { members. }\end{array}$ & $\begin{array}{l}\text { Based on } \\
\text { deJong- } \\
\text { Gierveld's } \\
\text { model of } \\
\text { loneliness, } \\
\text { rigorous } \\
\text { inclusion } \\
\text { criteria }\end{array}$ & $\begin{array}{l}\text { Small sample } \\
\text { size, } \\
\text { restricted to } \\
\text { small } \\
\text { geographic } \\
\text { area and } \\
\text { recruited by } \\
\text { single } \\
\text { pulmonologis } \\
\text { t }\end{array}$ \\
\hline $\begin{array}{l}\text { (Kramer, } \\
\text { Kapteyn, Kuik, \& } \\
\text { Deed, 2002) } \\
\text { Amsterdam, } \\
\text { Netherlands }\end{array}$ & $\begin{array}{l}\text { To determine } \\
\text { the association } \\
\text { between } \\
\text { hearing } \\
\text { impairment } \\
\text { and chronic } \\
\text { diseases, } \\
\text { including } \\
\text { stroke, and } \\
\text { psychosocial } \\
\text { status, } \\
\text { including } \\
\text { depression \& } \\
\text { loneliness }\end{array}$ & $\begin{array}{l}X \\
L\end{array}$ & $\begin{array}{l}\mathrm{N}=3,107 \text { in } \\
\text { Longitudinal } \\
\text { Aging Study } \\
\text { Amsterdam } \\
\text { (LASA) }\end{array}$ & $\begin{array}{l}\text { Depression - } \\
\text { Center for } \\
\text { Epidemiologic } \\
\text { Studies Depression } \\
\text { Scale (CES-D) } \\
\text { Self-efficacy - } \\
\text { General Self- } \\
\text { Efficacy Scale } \\
\text { Mastery - Pearlin } \\
\text { Mastery Scale } \\
\text { Loneliness - De } \\
\text { Jong-Gierveld } \\
\text { loneliness scale }\end{array}$ & $\mathrm{S}$ & $\begin{array}{l}\text { Those with stoke } \\
\text { impairment didn't } \\
\text { have statistically } \\
\text { significant rates of } \\
\text { loneliness while } \\
\text { those with hearing } \\
\text { impairment had } \\
\text { statistically } \\
\text { significant rates of } \\
\text { loneliness. }\end{array}$ & $\begin{array}{l}\text { One of only a } \\
\text { few studies that } \\
\text { measure the } \\
\text { effects of } \\
\text { psychosocial } \\
\text { outcomes in } \\
\text { relation to } \\
\text { hearing } \\
\text { problems } \\
\text { among persons } \\
\text { with chronic } \\
\text { conditions } \\
\text { including } \\
\text { stroke. }\end{array}$ & $\begin{array}{l}\text { Self-reported } \\
\text { variables - no } \\
\text { objective } \\
\text { measure of } \\
\text { hearing } \\
\text { impairment } \\
\text { or the } \\
\text { presence of } \\
\text { chronic } \\
\text { disease was } \\
\text { utilized }\end{array}$ \\
\hline $\begin{array}{l}\text { (Lofvenmark, } \\
\text { Mattiasson, } \\
\text { Billing, \& Edner, } \\
\text { 2009) } \\
\text { Sweden }\end{array}$ & $\begin{array}{l}\text { To investigate } \\
\text { perceived } \\
\text { loneliness and } \\
\text { social support } \\
\text { in patients with } \\
\text { HF }\end{array}$ & $X$ & $\mathrm{~N}=149$ & $\begin{array}{l}\text { Face to face } \\
\text { questionnaires }\end{array}$ & HD & $\begin{array}{l}\text { Loneliness } \\
\text { reported in } 29 \% \text { of } \\
\text { participants, more } \\
\text { often in women. } \\
\text { Those with } \\
\text { loneliness had } \\
\text { more days } \\
\text { hospitalized (p = } \\
.044) \text { and more } \\
\text { hospital } \\
\text { readmissions (p = } \\
.027 \text { ) despite not } \\
\text { having more } \\
\text { severe heart } \\
\text { failure. }\end{array}$ & $\begin{array}{l}\text { Homogeneous } \\
\text { sample of heart } \\
\text { failure patients }\end{array}$ & $\begin{array}{l}\text { Self-reported } \\
\text { variables }\end{array}$ \\
\hline
\end{tabular}


Table 1. Contd....

\begin{tabular}{|c|c|c|c|c|c|c|c|c|}
\hline $\begin{array}{c}\text { (Authors, Year) } \\
\text { Location }\end{array}$ & Aim & Design & Sample Size & Data Collection & CI & Results & Strengths & Weaknesses \\
\hline $\begin{array}{l}\text { (Momtaz, et al., } \\
\text { 2012) } \\
\text { Malaysia }\end{array}$ & $\begin{array}{l}\text { To examine } \\
\text { the impact of } \\
\text { loneliness on } \\
\text { hypertension } \\
\text { later in life }\end{array}$ & $\mathrm{X}$ & $\mathrm{N}=1,880$ & $\begin{array}{l}\text { Philadelphia } \\
\text { Geriatric Center } \\
\text { Morale Scale } \\
\text { (PGCMS) item, } \\
\text { "How much do you } \\
\text { feel lonely?" } \\
\text { Self-reported } \\
\text { chronic conditions }\end{array}$ & HD & $\begin{array}{l}\text { Older persons with } \\
\text { a lot of loneliness } \\
\text { had significantly } \\
\text { higher prevalence } \\
\text { of hypertension as } \\
\text { compared with } \\
\text { older persons with } \\
\text { low levels of } \\
\text { loneliness after } \\
\text { controlling for } \\
\text { sociodemographic } \\
\text { and health factors } \\
\text { [OR=1.31,(95\% CI } \\
1.04-1.66)]\end{array}$ & $\begin{array}{l}\text { Large } \\
\text { representative } \\
\text { sample of } \\
\text { elderly } \\
\text { Malaysian } \\
\text { population }\end{array}$ & 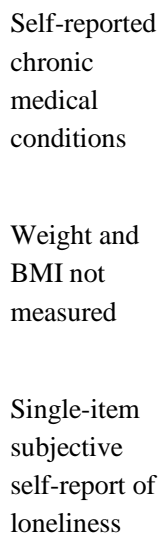 \\
\hline $\begin{array}{l}\text { (Nausheen, } \\
\text { Gidron, Gregg, } \\
\text { Tissarchondou, \& } \\
\text { Peveler, 2007) } \\
\text { UK }\end{array}$ & $\begin{array}{l}\text { To adapt an } \\
\text { implicit } \\
\text { measure of } \\
\text { loneliness, and } \\
\text { use it } \\
\text { alongside the } \\
\text { measures of } \\
\text { explicit } \\
\text { loneliness and } \\
\text { social support, }\end{array}$ & PPT & $\begin{array}{l}\mathrm{N}=25 \\
\text { women, 18- } \\
39 \text { years of } \\
\text { age }\end{array}$ & $\begin{array}{l}\text { Self-reported and } \\
\text { physical measures }\end{array}$ & $\mathrm{HD}$ & $\begin{array}{l}\text { Loneliness was } \\
\text { significantly } \\
\text { correlated with } \\
\text { diastolic blood } \\
\text { pressure reactivity } \\
\text { after one of the } \\
\text { stressors. }\end{array}$ & $\begin{array}{l}\text { Accurate } \\
\text { physical } \\
\text { measures }\end{array}$ & $\begin{array}{l}\text { Self-reported } \\
\text { measures }\end{array}$ \\
\hline
\end{tabular}


Table 1. Contd....

\begin{tabular}{|c|c|c|c|c|c|c|c|c|}
\hline $\begin{array}{c}\text { (Authors, Year) } \\
\text { Location }\end{array}$ & Aim & Design & Sample Size & Data Collection & CI & Results & Strengths & Weaknesses \\
\hline & $\begin{array}{l}\text { investigating } \\
\text { their } \\
\text { correlations } \\
\text { with } \\
\text { cardiovascular } \\
\text { reactivity to } \\
\text { lab stress }\end{array}$ & & & & & & & \\
\hline $\begin{array}{l}\text { (Nefs, Pouwer, } \\
\text { Pop, \& Denollet, } \\
\text { 2012) } \\
\text { Netherlands }\end{array}$ & $\begin{array}{l}\text { There were } 3 \\
\text { aims of this } \\
\text { study: to } \\
\text { examine the } \\
\text { validity of type } \\
\text { D personality } \\
\text { and its } \\
\text { assessment in } \\
\text { patients with } \\
\text { diabetes;clinic } \\
\text { al outcomes } \\
\text { correlated with } \\
\text { Type D } \\
\text { personality in } \\
\text { patients with } \\
\text { diabetes; Type } \\
\text { D personality } \\
\text { model across } \\
\text { men \& women } \\
\text { with diabetes. }\end{array}$ & PCD & $\begin{array}{l}\mathrm{N}=1553 \\
\text { primary care } \\
\text { patients with } \\
\text { type } 2 \\
\text { diabetes }\end{array}$ & $\begin{array}{l}\text { A single item was } \\
\text { used to measure } \\
\text { feelings of } \\
\text { loneliness in the } \\
\text { past } 12 \text { months } \\
\text { (ranging from } 1 \text { "I } \\
\text { never } \\
\text { felt lonely" to } 10 \text { "I } \\
\text { always felt } \\
\text { lonely"). }\end{array}$ & DM & $\begin{array}{l}\text { Patients with a } \\
\text { Type D personality } \\
\text { did not differ from } \\
\text { non-Type D } \\
\text { personality with } \\
\text { respect to diabetes } \\
\text { duration, } \\
\text { cardiovascular } \\
\text { disease history, } \\
\text { current } \\
\text { microvascular } \\
\text { complications or } \\
\text { physiological } \\
\text { cardiovascular risk } \\
\text { factors, including } \\
\text { glycemic control, } \\
\text { cholesterol and } \\
\text { blood pressure. } \\
\text { However, } \\
\text { participants with } \\
\text { Type D personality } \\
\text { reported more } \\
\text { loneliness, } \\
\text { emotional distress, } \\
\text { including } \\
\text { symptoms of } \\
\text { depressed mood, } \\
\text { anhedonia and } \\
\text { anxiety. Also, type } \\
\text { D women had a } \\
\text { more sedentary } \\
\text { lifestyle. }\end{array}$ & $\begin{array}{l}\text { The study } \\
\text { questions are } \\
\text { relevant and } \\
\text { the study adds } \\
\text { new } \\
\text { information } \\
\text { related to the } \\
\text { validity of } \\
\text { using a Type D } \\
\text { personality } \\
\text { inventory in } \\
\text { Diabetes } \\
\text { Patients. The } \\
\text { design is } \\
\text { appropriate for } \\
\text { the research } \\
\text { questions. The } \\
\text { statistical } \\
\text { analyses are } \\
\text { performed } \\
\text { correctly. }\end{array}$ & $\begin{array}{l}\text { Loneliness is } \\
\text { measured by } \\
\text { a single item } \\
\text { Likert scale } \\
\text { self-report } \\
\text { question. }\end{array}$ \\
\hline $\begin{array}{l}\text { (Norman et al., } \\
\text { 2011) } \\
\text { USA }\end{array}$ & $\begin{array}{l}\text { Examine the } \\
\text { role of } \\
\text { perceived } \\
\text { social isolation } \\
\text { in moderating } \\
\text { the effects of } \\
\text { oxytocin on } \\
\text { cardiac } \\
\text { autonomic } \\
\text { control in } \\
\text { humans. }\end{array}$ & E & $\begin{array}{l}\mathrm{N}=40 \\
20 \text { men and } \\
20 \text { women }\end{array}$ & $\begin{array}{l}\text { UCLA Loneliness } \\
\text { Scale } \\
\text { Beck Depression } \\
\text { Inventory } \\
\text { Perceived Social } \\
\text { Stress Scale (PSS- } \\
\text { 10) } \\
\text { Electrocardiogram } \\
\text { and impedance } \\
\text { cardiogram } \\
\text { Blood samples } \\
\text { Blood pressures }\end{array}$ & $\mathrm{HD}$ & $\begin{array}{l}\text { The effects of } \\
\text { oxytocin on } \\
\text { cardiac autonomic } \\
\text { control were } \\
\text { significantly } \\
\text { associated with } \\
\text { loneliness ratings. } \\
\text { Higher loneliness } \\
\text { was associated } \\
\text { with diminished } \\
\text { parasympathetic } \\
\text { cardiac reactivity } \\
\text { to intranasal } \\
\text { oxytocin. Thus, } \\
\text { lonely people may } \\
\text { be less responsive } \\
\text { to the effects of }\end{array}$ & $\begin{array}{l}\text { Randomized to } \\
\text { either the } \\
\text { oxytocin group } \\
\text { or placebo } \\
\text { group in a } \\
\text { double blind } \\
\text { manner }\end{array}$ & $\begin{array}{l}\text { Unable to } \\
\text { determine if } \\
\text { the effects of } \\
\text { oxytocin on } \\
\text { the } \\
\text { autonomic } \\
\text { nervous } \\
\text { system } \\
\text { activity are } \\
\text { isolated to } \\
\text { cardiac } \\
\text { output }\end{array}$ \\
\hline
\end{tabular}


Table 1. Contd....

\begin{tabular}{|c|c|c|c|c|c|c|c|c|}
\hline $\begin{array}{c}\text { (Authors, Year) } \\
\text { Location }\end{array}$ & Aim & Design & Sample Size & Data Collection & CI & Results & Strengths & Weaknesses \\
\hline & & & & & & $\begin{array}{l}\text { oxytocin on } \\
\text { cardiovascular } \\
\text { responsivity }\end{array}$ & & \\
\hline $\begin{array}{l}\text { (O'Donovan \& } \\
\text { Hughes, 2007) } \\
\text { Ireland }\end{array}$ & $\begin{array}{l}\text { Examine the } \\
\text { relation of } \\
\text { social support } \\
\text { at university } \\
\text { and loneliness } \\
\text { with pulse } \\
\text { pressure } \\
\text { reactivity to } \\
\text { acute } \\
\text { psychological } \\
\text { stress in } \\
\text { sample of } \\
\text { young adults }\end{array}$ & $\mathrm{X}$ & $\begin{array}{l}\mathrm{N}=65 \\
\text { college } \\
\text { females, } 17 \\
\text { to } 21 \text { years } \\
\text { of age }\end{array}$ & $\begin{array}{l}\text { Revised UCLA } \\
\text { Loneliness Scale. } \\
\text { Blood pressure } \\
\text { measurements } \\
\text { taken multiple } \\
\text { times at rest and } \\
\text { during stressor task. } \\
\text { The Social Support } \\
\text { at University Scale } \\
\text { (SSUS). }\end{array}$ & HD & $\begin{array}{l}\text { Students with } \\
\text { medium or high } \\
\text { loneliness had } \\
\text { significantly less } \\
\text { PP reactivity } \\
\text { compared to low } \\
\text { lonely students (P } \\
<.05) \text { for medium } \\
\text { and } .004 \text { for high } \\
\text { lonely }\end{array}$ & $\begin{array}{l}\text { UCLA scale } \\
\text { and took } \\
\text { physical } \\
\text { measures, used } \\
\text { calculations of } \\
\text { obtained blood } \\
\text { pressures to get } \\
\text { pulse pressure }\end{array}$ & $\begin{array}{l}\text { Small sample } \\
\text { size. } \\
\text { No male } \\
\text { students in } \\
\text { sample and } \\
\text { thus gender } \\
\text { difference for } \\
\text { loneliness } \\
\text { and pulse } \\
\text { pressure } \\
\text { could not be } \\
\text { assessed }\end{array}$ \\
\hline $\begin{array}{l}\text { (Österberg, Baigi, } \\
\text { Bering, \& } \\
\text { Fridlund, 2010) } \\
\text { Sweden }\end{array}$ & $\begin{array}{l}\text { To explore } \\
\text { perceived } \\
\text { importance and } \\
\text { knowledge of } \\
\text { known risk } \\
\text { factors for } \\
\text { coronary artery } \\
\text { disease among } \\
\text { non-attendees } \\
\text { in cardiac } \\
\text { rehab } \\
\text { programs }\end{array}$ & DC & $\begin{array}{l}106 \text { patients } \\
\text { Treated for } \\
\text { myocardial } \\
\text { infarction } \\
\text { (MI) and/or } \\
\text { coronary } \\
\text { artery bypass } \\
\text { graft } \\
\text { (CABG) or } \\
\text { cardiac cath. } \\
\text { and who } \\
\text { declined to } \\
\text { participate in } \\
\text { cardiac } \\
\text { rehabilitation } \\
69 \% \text { men }\end{array}$ & $\begin{array}{l}\text { Self-report } \\
\text { questionnaires }\end{array}$ & HD & $\begin{array}{l}\text { Participants who } \\
\text { were declining } \\
\text { cardiac rehab were } \\
\text { aware that } \\
\text { psychosocial } \\
\text { factors contributed } \\
\text { to heart disease. }\end{array}$ & $\begin{array}{l}\text { No comparison } \\
\text { group - those } \\
\text { who attend } \\
\text { cardiac } \\
\text { rehabilitation }\end{array}$ & $\begin{array}{l}\text { Newly } \\
\text { developed } \\
\text { questionnaire } \\
\text { has not been } \\
\text { tested for } \\
\text { reliability }\end{array}$ \\
\hline $\begin{array}{l}\text { (Patterson \& } \\
\text { Veenstra, 2010) } \\
\text { USA }\end{array}$ & $\begin{array}{l}\text { To investigate } \\
\text { the impact of } \\
\text { loneliness on } \\
\text { all-cause } \\
\text { mortality, } \\
\text { mortality from } \\
\text { ischemic } \\
\text { disease, and } \\
\text { mortality from } \\
\text { other } \\
\text { cardiovascular } \\
\text { diseases. }\end{array}$ & $\mathrm{L}$ & $\begin{array}{l}\mathrm{N}=6,768 \text { in } \\
1965 ; \\
\mathrm{N}=4,522 \text { in } \\
1999 \\
\\
3,168 \text { of } \\
\text { those in the } \\
\text { original } \\
\text { sample died } \\
\text { by the end of } \\
\text { the study }\end{array}$ & $\begin{array}{l}\text { Alameda County } \\
\text { Health and Ways of } \\
\text { Living Study } \\
\text { Self-reported } \\
\text { measure in } \\
\text { response to } \\
\text { question about how } \\
\text { often they feel } \\
\text { "very lonely or } \\
\text { remote from other } \\
\text { people" with } \\
\text { response categories } \\
\text { of never, sometimes } \\
\text { and often } \\
\text { Death certificate } \\
\text { data }\end{array}$ & HD & $\begin{array}{l}\text { Frequent loneliness } \\
\text { was not } \\
\text { significantly } \\
\text { associated with } \\
\text { mortality from } \\
\text { ischemic heart } \\
\text { disease but it more } \\
\text { than doubled the } \\
\text { odds of mortality } \\
\text { from other } \\
\text { ailments of the } \\
\text { circulatory system } \\
\text { in models } \\
\text { controlling for age } \\
\text { and gender. } \\
\text { Chronic or recent } \\
\text { loneliness may } \\
\text { increase risk for } \\
\text { mortality }\end{array}$ & $\begin{array}{l}\text { One of only a } \\
\text { few } \\
\text { longitudinal } \\
\text { studies on a } \\
\text { representative } \\
\text { sample of the } \\
\text { health effects } \\
\text { on loneliness }\end{array}$ & $\begin{array}{l}\begin{array}{l}\text { Self-reported } \\
\text { data }\end{array} \\
\text { Imprecise } \\
\text { measure of } \\
\text { loneliness } \\
\text { Irregular } \\
\text { intervals of } \\
\text { data } \\
\text { collection } \\
\text { Small sample } \\
\text { size for some } \\
\text { causes of } \\
\text { death (i.e. } \\
\text { suicide) other } \\
\text { than } \\
\text { cardiovascula } \\
\text { r deaths }\end{array}$ \\
\hline
\end{tabular}


Table 1. Contd....

\begin{tabular}{|c|c|c|c|c|c|c|c|c|}
\hline $\begin{array}{c}\text { (Authors, Year) } \\
\text { Location }\end{array}$ & Aim & Design & Sample Size & Data Collection & CI & Results & Strengths & Weaknesses \\
\hline $\begin{array}{l}\text { (Penninx et al., } \\
\text { 1999) } \\
\text { Amsterdam, } \\
\text { Netherlands }\end{array}$ & $\begin{array}{l}\text { To explore } \\
\text { social network } \\
\text { size, functional } \\
\text { social support } \\
\text { and loneliness } \\
\text { among elderly } \\
\text { with different } \\
\text { chronic } \\
\text { diseases }\end{array}$ & $X, L$ & $\begin{array}{l}\mathrm{N}=3,107 \text { in } \\
\text { Longitudinal } \\
\text { Aging Study } \\
\text { Amsterdam } \\
\text { (LASA) } \\
\mathrm{N}=2,788 \\
\text { included in } \\
\text { sample for } \\
\text { this study }\end{array}$ & $\begin{array}{l}\text { De Jong Gierveld } \\
\text { loneliness scale }\end{array}$ & S & $\begin{array}{l}\text { Among all the } \\
\text { chronic diseases } \\
\text { studied including } \\
\text { stroke, only } \\
\text { peripheral vascular } \\
\text { disease, lung } \\
\text { disease and } \\
\text { arthritis were } \\
\text { found to have risks } \\
\text { for feelings of } \\
\text { loneliness. }\end{array}$ & $\begin{array}{l}\text { Comparisons } \\
\text { of people with } \\
\text { and without } \\
\text { different } \\
\text { chronic } \\
\text { diseases in } \\
\text { their social } \\
\text { networks, } \\
\text { social support } \\
\text { and loneliness } \\
\text { patterns }\end{array}$ & $\begin{array}{l}\text { High } \\
\text { nonresponse } \\
\text { rate among } \\
\text { oldest age } \\
\text { groups which } \\
\text { are } \\
\text { associated } \\
\text { with ill } \\
\text { physical } \\
\text { health and } \\
\text { this may } \\
\text { cause a } \\
\text { weakening of } \\
\text { the } \\
\text { associations } \\
\text { explored }\end{array}$ \\
\hline $\begin{array}{l}\text { (Seo, Yates, } \\
\text { Dizona, } \\
\text { Laframboise, \& } \\
\text { Norman, 2014) } \\
\text { USA }\end{array}$ & $\begin{array}{l}\text { To enhance } \\
\text { understanding } \\
\text { of the effects } \\
\text { of depression } \\
\text { on patients } \\
\text { with heart } \\
\text { failure }\end{array}$ & $\mathrm{X}, \mathrm{DC}$ & $\begin{array}{l}\mathrm{N}=150 \\
\text { Adults }\end{array}$ & & $\mathrm{HD}$ & $\begin{array}{l}\text { In patients with } \\
\text { heart failure, those } \\
\text { who were lonely } \\
\text { had more dyspnea. }\end{array}$ & $\begin{array}{l}\text { Cross- } \\
\text { sectional, used } \\
\text { reliable and } \\
\text { valid } \\
\text { instruments. } \\
\text { Used SEM to } \\
\text { demonstrate } \\
\text { relationships }\end{array}$ & $\begin{array}{l}\text { No causality } \\
\text { established }\end{array}$ \\
\hline $\begin{array}{l}\text { (Smith, Theeke, } \\
\text { Culp, Clark, \& } \\
\text { Pinto, 2014) } \\
\text { USA } \\
\text { (West Virginia) }\end{array}$ & $\begin{array}{l}\text { To describe } \\
\text { relationships } \\
\text { among self- } \\
\text { rated health, } \\
\text { stress, sleep } \\
\text { quality, } \\
\text { loneliness, and } \\
\text { self-esteem, in } \\
\text { obese young } \\
\text { adult women }\end{array}$ & $\mathrm{X}$ & $\begin{array}{l}\mathrm{N}=68 \\
\text { (BMI } 30 \text { or } \\
\text { higher; age } \\
\text { 18-34 years) }\end{array}$ & $\begin{array}{l}\text { Demographics - } \\
\text { Age \& BMI } \\
\text { Self-rated health - } \\
5 \text { point Likert scale, } \\
\text { then dichotomized } \\
\text { for } 2 \text { groups: } \\
\text { poor/fair and } \\
\text { good/very good. } \\
\text { Perceived stress } \\
\text { scale,10-item tool } \\
\text { Revised UCLA } \\
\text { loneliness scale. } \\
\text { Rosenberg Self- } \\
\text { esteem Scale. } \\
\text { Pittsburgh Sleep } \\
\text { Quality Index, } 19 \\
\text { self-rated items. }\end{array}$ & $\mathrm{O}$ & $\begin{array}{l}\text { Higher perceived } \\
\text { stress had a } \\
\text { stronger positive } \\
\text { correlation with } \\
\text { both increased } \\
\text { loneliness ( } r= \\
0.560, p<.001) \\
\text { and poorer sleep } \\
\text { quality }(r=0.414, \\
p<.001) . \\
\text { Loneliness was } \\
\text { negatively related } \\
\text { to self-esteem }(r= \\
-0.688, p<.001) . \\
\text { Participants who } \\
\text { rated health as } \\
\text { poor/fair reported } \\
\text { higher mean scores } \\
\text { for stress }(M= \\
23.25) \text { and } \\
\text { loneliness }(M= \\
47.20) \text { and, lower } \\
\text { mean scores for } \\
\text { self-esteem }(M= \\
15.65) \text { when } \\
\text { compared to those } \\
\text { who rated health as } \\
\text { good/very good }(M \\
=17.42, M= \\
37.10, \text { and } M= \\
22.73) .\end{array}$ & $\begin{array}{l}\text { Enhances } \\
\text { understanding } \\
\text { of the } \\
\text { relationship } \\
\text { between stress, } \\
\text { sleep, } \\
\text { loneliness, self- } \\
\text { esteem and } \\
\text { self-rated } \\
\text { health status } \\
\text { among college } \\
\text { students }\end{array}$ & $\begin{array}{l}\text { Convenience } \\
\text { sample. }\end{array}$ \\
\hline
\end{tabular}


Table 1. Contd....

\begin{tabular}{|c|c|c|c|c|c|c|c|c|}
\hline $\begin{array}{l}\text { (Authors, Year) } \\
\text { Location }\end{array}$ & Aim & Design & Sample Size & Data Collection & CI & Results & Strengths & Weaknesses \\
\hline $\begin{array}{l}\text { (Taylor, 2009) } \\
\text { Canada }\end{array}$ & $\begin{array}{l}\text { To evaluation } \\
\text { the } \\
\text { effectiveness } \\
\text { of } \\
\text { videoconferenc } \\
\text { ing for an } \\
\text { educational } \\
\text { and exercise } \\
\text { self- } \\
\text { management } \\
\text { program for } \\
\text { people who } \\
\text { have had a } \\
\text { stroke and } \\
\text { their caregivers }\end{array}$ & $\mathrm{L}, \mathrm{E}$ & $\begin{array}{l}\mathrm{N}=12 \text { people } \\
\text { with stroke } \\
\mathrm{N}=4 \\
\text { caregiver }\end{array}$ & $\begin{array}{l}\text { Focus groups, } \\
\text { attendance rates, } \\
\text { Geriatric } \\
\text { Depression Scale } \\
\text { (GDS), Activity- } \\
\text { Specific Balance } \\
\text { Confidence Scale, } \\
\text { Berg Balance Scale, } \\
\text { 6-Minute Walk } \\
\text { Test, Goal } \\
\text { Attainment Scaling }\end{array}$ & S & $\begin{array}{l}\text { Mean depression } \\
\text { scores on GDS } \\
\text { ranged from pre- } \\
\text { test }(\mathrm{M}=5.3 \text {, } \\
\mathrm{SD}=3.2) \text {, post-test } \\
(\mathrm{M}=4.9, \mathrm{SD}=2.1) \\
\text { to } 3 \text {-month follow- } \\
\text { up }(\mathrm{M}=3.2 \text {, } \\
\mathrm{SD}=2.3)\end{array}$ & $\begin{array}{l}\text { Intervention - } \\
\text { experimental } \\
\text { design }\end{array}$ & $\begin{array}{l}\text { No specific } \\
\text { measure of } \\
\text { loneliness }\end{array}$ \\
\hline $\begin{array}{l}\text { (Theeke \& } \\
\text { Mallow, 2013) } \\
\text { USA }\end{array}$ & $\begin{array}{l}\text { To learn more } \\
\text { about the } \\
\text { prevalence of } \\
\text { loneliness in } \\
\text { rural older } \\
\text { adults with } \\
\text { chronic illness. }\end{array}$ & $\mathrm{X}$ & $\begin{array}{l}60 \text { adults age } \\
65 \text { and older }\end{array}$ & $\begin{array}{l}\text { Face to face } \\
\text { interviews and } \\
\text { EHR record review }\end{array}$ & $\mathrm{HD}$ & $\begin{array}{l}\text { Participants with } \\
\text { heart disease had } \\
\text { significantly higher } \\
\text { loneliness scores } \\
\text { than those without }\end{array}$ & $\begin{array}{l}\text { Cross-sectional } \\
\text { descriptive } \\
\text { design using } \\
\text { valid and } \\
\text { reliable } \\
\text { instruments }\end{array}$ & $\begin{array}{l}\text { Only } \\
\text { descriptive, } \\
\text { self-reported } \\
\text { variables }\end{array}$ \\
\hline $\begin{array}{l}\text { (Theeke et al., } \\
\text { 2014) } \\
\text { USA }\end{array}$ & & & & & S & & & \\
\hline $\begin{array}{l}\text { (Tigani, } \\
\text { Artemiadis, } \\
\text { Alexopoulos, } \\
\text { Chrousos, \& } \\
\text { Darviri, 2012) } \\
\text { Greece }\end{array}$ & $\begin{array}{l}\text { To study } \\
\text { associations of } \\
\text { various } \\
\text { sociodemograp } \\
\text { hic, disease- } \\
\text { related, } \\
\text { lifestyle \& } \\
\text { psychosocial } \\
\text { factors, ability } \\
\text { to perform } \\
\text { activities of } \\
\text { daily living } \\
\text { with self-rated } \\
\text { health (SRH) } \\
\text { in } \\
\text { centenarians. } \\
\text { Lifestyle } \\
\text { factors - } \\
\text { smoking, BMI, } \\
\text { sleep } \\
\text { satisfaction. }\end{array}$ & $\mathrm{X}$ & $\begin{array}{l}\mathrm{N}=400 \\
\text { (adults } 100- \\
109 \text { years) }\end{array}$ & $\begin{array}{l}\text { Self-rated health- } \\
\text { single item with } \\
\text { Likert scale answer } \\
\text { Classified into } \\
\text { healthy, one } \\
\text { disease, or co- } \\
\text { morbidity. } \\
\text { Loneliness - "Do } \\
\text { you feel lonely or } \\
\text { abandoned?" } \\
\text { International } \\
\text { Personality Item } \\
\text { Pool (IPIP) } \\
\text { Health Locus of } \\
\text { Control (HLC) }\end{array}$ & $\mathrm{O}$ & $\begin{array}{l}\text { Nearly half were } \\
\text { normal weight } \\
(48.5 \%) \text { and more } \\
\text { than a third were } \\
\text { overweight or } \\
\text { obese (35.1\%). } \\
\text { Significant } \\
\text { relationship } \\
\text { between worse } \\
\text { Self-Rated health } \\
\text { and feelings of } \\
\text { loneliness (p<0.01) } \\
\text { Obese subjects } \\
\text { reported very good } \\
\text { SRH more often } \\
\text { than individuals } \\
\text { with normal BMI } \\
\text { (OR, 95\% CI: } \\
\text { 5.35, 1.67-17.18), }\end{array}$ & $\begin{array}{l}\text { Large sample } \\
\text { size } \\
\text { Identification } \\
\text { of multiple } \\
\text { non-disease } \\
\text { factors that } \\
\text { influence SRH. }\end{array}$ & $\begin{array}{l}\text { Disease } \\
\text { status - one } \\
\text { item "Do you } \\
\text { currently } \\
\text { suffer from a } \\
\text { condition } \\
\text { requiring } \\
\text { regular } \\
\text { medical } \\
\text { treatment?" } \\
\text { Mis-match in } \\
\text { data from } \\
\text { tables and } \\
\text { text, so } \\
\text { unsure which } \\
\text { is correct. } \\
\\
\text { Results may } \\
\text { not be } \\
\text { generalizable }\end{array}$ \\
\hline
\end{tabular}


Table 1. Contd....

\begin{tabular}{|c|c|c|c|c|c|c|c|c|}
\hline $\begin{array}{l}\text { (Authors, Year) } \\
\text { Location }\end{array}$ & Aim & Design & Sample Size & Data Collection & CI & Results & Strengths & Weaknesses \\
\hline & $\begin{array}{l}6 \text { questions of } \\
\text { food } \\
\text { consumption }\end{array}$ & & & & & $\begin{array}{l}\text { but this was not the } \\
\text { case for good } v s \text {. } \\
\text { poor comparisons } \\
\text { of loneliness and } \\
\text { pessimism, which } \\
\text { were significantly } \\
\text { related with worse } \\
\text { SRH for both } \\
\text { comparison groups } \\
\text { (OR, 95\% CI: } 2.23 \\
(1.24-4.01) \text { and } \\
(\text { OR, } 95 \% \text { CI: } 2.15 \\
(1.22-3.8) \text {. }\end{array}$ & & $\begin{array}{l}\text { to other age } \\
\text { groups. } \\
\text { Measurement } \\
\text { s mainly } \\
\text { based on } \\
\text { self-reporting } \\
\text { Non-random } \\
\text { sampling }\end{array}$ \\
\hline $\begin{array}{l}\text { (Tomaka, } \\
\text { Thompson, \& } \\
\text { Palacios, 2006) } \\
\text { USA } \\
\text { New Mexico }\end{array}$ & $\begin{array}{l}\text { To examine } \\
\text { relationships } \\
\text { among social } \\
\text { isolation, } \\
\text { loneliness, and } \\
\text { social support } \\
\text { to health } \\
\text { outcomes }\end{array}$ & $\mathrm{X}$ & $\begin{array}{l}\mathrm{N}=755 \\
60 \text { years of } \\
\text { age and older }\end{array}$ & $\begin{array}{l}\text { UCLA Loneliness } \\
\text { scale }\end{array}$ & $\begin{array}{c}\mathrm{HD}, \\
\mathrm{S}\end{array}$ & $\begin{array}{l}\text { Belongingness } \\
\text { support is needed } \\
\text { and related most } \\
\text { consistently to } \\
\text { health outcomes }\end{array}$ & $\begin{array}{l}\text { Survey face to } \\
\text { face }\end{array}$ & $\begin{array}{l}\text { Cross- } \\
\text { sectional and } \\
\text { self-reported } \\
\text { data } \\
\text { Uncertain } \\
\text { cultural } \\
\text { appropriatene } \\
\text { ss of the } \\
\text { support and } \\
\text { loneliness } \\
\text { measures }\end{array}$ \\
\hline $\begin{array}{l}\text { (Wågert et al., } \\
\text { 2005) } \\
\text { Sweden }\end{array}$ & $\begin{array}{l}\text { To explore } \\
\text { social, } \\
\text { functional, and } \\
\text { medical factors } \\
\text { associated with }\end{array}$ & $\mathrm{X}, \mathrm{O}$ & $\begin{array}{l}\mathrm{N}=319 \\
85 \text { years and } \\
\text { older }\end{array}$ & $\begin{array}{l}\text { Philadelphia } \\
\text { Geriatric Center } \\
\text { Morale Scale } \\
\text { (PGCMS), Barthel } \\
\text { Activities of Daily }\end{array}$ & S & $\begin{array}{l}\text { Participants who } \\
\text { did not feel lonely, } \\
\text { living in ordinary } \\
\text { housing, not } \\
\text { having depression }\end{array}$ & $\begin{array}{l}\text { Large } \\
\text { randomized } \\
\text { sample }\end{array}$ & $\begin{array}{l}\text { No direct } \\
\text { measure of } \\
\text { loneliness in } \\
\text { stroke } \\
\text { patients }\end{array}$ \\
\hline
\end{tabular}


Table 1. Contd....

\begin{tabular}{|c|c|c|c|c|c|c|c|c|}
\hline $\begin{array}{c}\text { (Authors, Year) } \\
\text { Location }\end{array}$ & Aim & Design & Sample Size & Data Collection & CI & Results & Strengths & Weaknesses \\
\hline & $\begin{array}{l}\text { morale among } \\
\text { elderly }\end{array}$ & & & $\begin{array}{l}\text { Living Index, } \\
\text { Geriatric } \\
\text { Depression Scale, } \\
\text { Mini-Mental State } \\
\text { Examination, Mini } \\
\text { Nutritional } \\
\text { Assessment and } \\
\text { symptom } \\
\text { questionnaire }\end{array}$ & & $\begin{array}{l}\text { and with self-rated } \\
\text { good to excellent } \\
\text { health had } \\
\text { significantly higher } \\
\text { PGCMS scores } \\
(\mathrm{p}<0.001)\end{array}$ & & $\begin{array}{l}\text { Cross- } \\
\text { sectional } \\
\text { limitations } \\
\text { Oldest age } \\
\text { groups had } \\
\text { some } \\
\text { cognitive } \\
\text { impairment } \\
\text { and couldn't } \\
\text { answer the } \\
\text { PGCMS }\end{array}$ \\
\hline
\end{tabular}


Table 1. Contd....

\begin{tabular}{|c|c|c|c|c|c|c|c|c|}
\hline $\begin{array}{c}\text { (Authors, Year) } \\
\text { Location }\end{array}$ & Aim & Design & Sample Size & Data Collection & CI & Results & Strengths & Weaknesses \\
\hline & & & & & & $\begin{array}{l}\text { circumference }(p \\
<.001, \mathrm{OR}=1.1, \\
95 \% \mathrm{CI}=1.0,1.2) .\end{array}$ & $\begin{array}{l}\text { Large sample } \\
\text { size }\end{array}$ & $\begin{array}{l}\text { syndrome } \\
\text { over time. }\end{array}$ \\
\hline $\begin{array}{l}\text { Wyller, Holmen, } \\
\text { Laake, \& Laake, } \\
\text { (1998) } \\
\text { Norway }\end{array}$ & $\begin{array}{l}\text { To explore the } \\
\text { subjective } \\
\text { perspective of } \\
\text { well-being } \\
\text { among people } \\
\text { who have had } \\
\text { a stroke }\end{array}$ & $\begin{array}{c}\mathrm{X} \\
\\
\text { two } \\
\text { sample } \\
\text { factor } \\
\text { analysi } \\
\mathrm{s} \\
\text { model }\end{array}$ & $\begin{array}{l}\mathrm{N}=1417 \\
\text { stroke } \\
\text { patients } \\
\mathrm{N}=1439 \\
\text { comparison } \\
\text { group of } \\
\text { stroke-free } \\
\text { people } \\
\text { Sample from } \\
\text { cross- } \\
\text { sectional } \\
\text { study } \\
\mathrm{N}=74,977\end{array}$ & $\begin{array}{l}\text { Nord-Trondelag } \\
\text { Health Survey - } \\
\text { Height, weight, } \\
\text { blood pressure, } \\
\text { blood glucose. } \\
\text { Questionnaires on } \\
\text { perceived health, } \\
\text { functional abilities, } \\
\text { contact with health } \\
\text { care system, work } \\
\text { conditions, chronic } \\
\text { disease, lifestyle, } \\
\text { housing, education } \\
\text { level, medical } \\
\text { symptoms, social } \\
\text { support, subjective } \\
\text { well-being (SWB) }\end{array}$ & S & $\begin{array}{l}\text { A fitted linear } \\
\text { regression model } \\
\text { with } 12 \\
\text { explanatory } \\
\text { variables including } \\
\text { loneliness } \\
\text { explained } 50.3 \% \text { of } \\
\text { the variance in the } \\
\text { SWB score. }\end{array}$ & $\begin{array}{l}\text { Large } \\
\text { randomized } \\
\text { sample size }\end{array}$ & $\begin{array}{l}\text { Concurrent } \\
\text { validity of a } \\
\text { measure of } \\
\text { the SWB } \\
\text { could not be } \\
\text { established } \\
\text { because a } \\
\text { gold standard } \\
\text { does not } \\
\text { exist. } \\
\text { Cross- } \\
\text { sectional } \\
\text { design } \\
\text { doesn't allow } \\
\text { for the effect } \\
\text { of age on } \\
\text { SWB to be } \\
\text { measured }\end{array}$ \\
\hline
\end{tabular}

Authors: APA format

Year: Year of Publication

Aim: Purpose of the Study

Study Design: Cross-sectional (X), Descriptive Correlational (DC), Experimental (E), Mixed methods (M), Quasi-experimental (QE) , Retrospective (R), Longitudinal (L), Observational (O), Pre-Post Test (PPT), Prospective cohort design (PCD)

Sample Size: $\mathrm{n}$ and target population

Data Collection: Self-report questionnaires, observations, physiological, medical records

Chronic Disease: Diabetes (DM); Heart Disease (HD); Obesity (O); Pulmonary Disease (P); Stroke (S);

\section{LONELINESS AND CARDIOVASCULAR HEALTH PROBLEMS}

Three studies examined relationships among loneliness and overall diagnosis of heart conditions. [14-16]. Tomaka (2006) studied 755 older adults and concluded that loneliness is prevalent and that belongingess support could result in improved health outcomes. Theeke and Mallow (2013) reported that loneliness is prevalent in a sample of 60 older adults with multiple chronic conditions including heart disease and that those with heart disease had some of the highest loneliness scores. Finally, Patterson and Veenstra (2010) investigated the impact of loneliness on all-cause mortality and concluded that loneliness significantly increased the odds of death from cardiovascular related problems.

\section{LONELINESS, CARDIOVASCULAR REACTIVITY, AND HYPERTENSION}

Six different studies focused on the relationships among loneliness, cardiovascular reactivity, and blood pressure [12, 17-21]. These studies provide evidence, in samples that ranged from young adults to older adults, that loneliness is related to cardiovascular reactivity [17] and demonstrate that lonely adult caregivers have greater activation of the sympathetic branch of the autonomic nervous system when compared to those less lonely, and that lonely people have higher total peripheral resistance [18]. Nausheen et al. (2007) studied women between ages of 18 and 29 years and reported that loneliness correlated with diastolic blood pressure reactivity. O'Donovan and Hughes (2007) studied young adult females and concluded that those with medium or high loneliness scores had significantly less pulse pressure reactivity when compared to those with low loneliness. Norman et al. (2011) studied the effects of oxytocin on cardiac autonomic control and evaluated the influence of loneliness on this response to oxytocin in a sample of 40 adults (20 women and 20 men). In this study, it was demonstrated that loneliness predicted changes in respiratory and cardiovascular function when compared to a placebo group. This finding is significant because this change existed even while controlling for independent influence of depression, anxiety, and other serum lab values, leading to the conclusion that loneliness may actually create a change in normal autonomic response to a health diminishing response. Finally, Momtaz et al. (2012) studied 1,880 older Maylasians and concluded that loneliness significantly increased the likelihood of hypertension in later life (OR $1.31, \mathrm{p}<.05,95 \%$, CI 1.04-1.66).

\section{LONELINESS AND CORONARY HEART DISEASE}

Three studies focused on relationships among loneliness and the development or treatment of those with coronary 
heart disease (CHD). [22-24]. In 2009, Thurston and Kubzansky reported findings from a data analyses of existing National Health and Nutrition Study (NHANES). Loneliness is assessed in this data using the single self-report item from the Center for Epidemiological Studies Depression Scale (CES-D). Based on these analyses, adults with high loneliness had higher risk of having coronary heart disease. Tobo-Medina and Canaval-Erazo (2010) identified loneliness as one of 10 predominant characteristics in a sample of 65 men and women with CHD. Finally, in 106 patients who had already been treated for myocardial infarction and were expected to enroll in cardiac rehabilitation services, it was recognized that the participants were unaware that loneliness could have significantly contributed to their CHD, indicating a need for education in this specific group.

\section{LONELINESS AND HEART FAILURE}

Three studies of heart failure patients indicated that loneliness is also problematic in this population. In one sample of 149 heart failure patients, loneliness was identified as a prevalent problem (29\%) [25]. In addition, in this same heart failure population, loneliness was associated with increased healthcare utilization including more days hospitalized and more re-hospitalizations. [25]. In a more recent cross-sectional study of 150 adults with heart failure, participants with loneliness expressed significantly more dyspnea [26]. Finally, in a recent longitudinal study of loneliness and biomarkers of heart failure disease severity (sample included 268 adults experiencing heart failure), loneliness was independently associated with higher depression levels at 1-year follow-up $(p$ < .05) [27].

\section{LONELINESS AND ISCHEMIC STROKE}

Twenty-nine articles were initially retrieved in the literature search on loneliness and stroke. Nineteen of the articles were eliminated as follows: 8 were studies of caregivers; 5 were qualitative; 3 were poster abstracts; 1 didn't include a stroke measurement; 1 was a commentary; and 1 was a duplicate of an article reviewed for the diabetes section of this paper. Therefore, 10 studies were included that measured loneliness in a sample of people who had suffered stroke.

Loneliness has been associated with increased prevalence of stroke and increased mortality, disability, and poor recovery following stroke [28-30]. Stroke patients reported higher perceived social isolation when compared to agematched healthy individuals [31]; and loneliness was linked to an increased risk of post-stroke depression (PSD). PSD encompasses a broad class of emotional stressors, including depression, depressive-like symptoms, mood disorders, anxiety, and loneliness and is associated with increased mortality, stroke recurrence, disability, and decreased quality of life. Duration and time of onset of PSD are variable, lasting as few as several weeks in some, or persisting longer than 20 months in others [32]. Regardless of the time of onset or the duration of PSD, the effect on stroke recovery is striking. Consensus from these studies is that the loneliness and depression have an increased prevalence in stroke survivors, and that both are associated with decreased overall quality of life.

Several factors were identified that influence and predict the degree of loneliness and depression among stroke survivors, the most significant being presence of loneliness or depression prior to the stroke, stroke severity, and whether or not the patient went to a nursing or rehabilitation facility. Nine of the 10 studies reported an increases depression and loneliness and decreased quality of life, well-being, and morale among stroke survivors compared to controls [14, 3342]. Two studies reported that depression and loneliness were higher among stroke patients discharged to a nursing home or other care facility in comparison to those discharged home [33] (Jongenelis, 2004). Similarly, Wagert (2005) reported that stroke survivors living at home alone reported increased feelings of loneliness compared to age-matched controls living at home alone. In addition, Wagert (2005) reported that stroke survivors who lived at home, but were not alone, had higher morale compared to age-matched older adults who had not suffered a stroke. These findings suggest that social support in a familiar home environment is associated with a greater quality of life and morale, even in the absence of stroke.

Penninx (1999) reported contradictory findings concerning discharge location and loneliness. Penninx et al. interviewed 2,788 elderly citizens in the Netherlands, both stroke survivors and non-stroke controls, to assess feelings of loneliness, as part of the larger Longitudinal Aging Study Amsterdam (LASA) [43]. This group reported that stroke survivors received more social and instrumental support compared to other chronic diseases, and consequently, stroke survivors were not at a higher risk of loneliness than nonstroke controls. These contradictory findings may be the result of differing studying design, or cultural differences in the populations under study. Penninx (1999) excluded participants living in nursing homes because social support in the long-term care facilities was incomparable to older adults who are living independently. This exclusion criterion makes the findings of Penninx (1999) incomparable to that of Theeke and Barr (2014) and Jongenelis (2004). Further, Penninx (1999) used the de Jong Gierveld loneliness scale instead of the more widely used UCLA Loneliness Scale (as used in both Theeke and Barr (2014) and Jongenelis (2004)), which may also account for the discrepant findings.

There are several other factors that have been shown to mediate loneliness in stroke, including socioeconomic status [33], sensory deficits following stroke [38, 39, 41], and race [14]. Specifically, Theeke and Barr (2014) reported higher feelings of loneliness and decreased quality of life in stroke survivors in the Appalachian region of the United States compared to more urban areas of the US. This region is associated with a lower median household income than the national average that is consistently associated with negative health outcomes in a variety of disease states. It is also important to consider that many inhabitants of the Appalachian region populate rural areas that are geographically isolated from health care facilities. Both of these factors may contribute to the increased loneliness, depression, and incidence of stroke in this region, and these findings may extend to other rural underserved populations. 
The correlation between socioeconomic status and loneliness after stroke may be related to race or ethnicity. Tomaka (2006) found that while loneliness is a predictor of stroke outcome in Hispanics, loneliness does not predict stroke outcome in a Caucasian population. As national data suggests that the US Hispanic population has a lower socioeconomic status than Caucasians, these findings may reflect confounding differences in known determinants of health experienced by diverse populations

The goal of all of these studies was to describe loneliness, depression, and quality of life following stroke and identify the factors that mediate these conditions, with the ultimate goal of developing clinical care standards that will improve stroke prognosis by reducing loneliness and depression. Of the ten studies described, only one focused on progressing clinical care. Taylor (2009) described the implementation of a videoconference, telehealth program for stroke survivors and their caregivers in a rural population. This community program titled, Moving On After Stroke (MOST), was delivered to 12 stroke survivors in a rural population. MOST is a 9-week program with two, two-hour sessions per week for subjects. These weekly sessions consisted of one hour of discussion, followed by one hour of physical exercise. After the 9-week MOST program, Taylor (2009) reported that both stroke survivors and caregivers reported lower loneliness scores when compared to subjects who had not undergone MOST. Given the findings of Theeke and Barr (2014), the MOST program may provide a means to provide social support to rural populations and reduce loneliness and improve stroke prognosis.

\section{LONELINESS AND OBESITY}

Of the 17 articles identified on loneliness and obesity, only 7 were included in this review. Three were excluded because they didn't include a measure of loneliness, 2 were excluded because they were reviews, 1 was excluded because it didn't include a measure of obesity, 1 was excluded because it focused on child subjects, 1 was a commentary, 1 was a duplicate and another was excluded because it was a sensitivity analysis. Obesity and loneliness are conditions that both negatively impact health. An association between obesity and loneliness exists [44-48]. However, the exact nature and extent of the relationship between obesity and loneliness is unclear and warrants further study.

Obesity and loneliness appear to have a synergistic negative impact on both physical and emotional health. For example, Jacobs et al. (2006) found loneliness was associated with poor sleep ratings, and that poor sleep ratings were associated with obesity, low physical activity, depression and other negative health outcomes. Additionally, increased loneliness was seen in those with increased weight-related stigma which includes lower rates of hire and promotion, higher rates of wrongful dismissal and social isolation [44]. Morse, et al. (2006) studied night time eating symptoms and found a relationship between eating in response to emotions including anger, sadness, loneliness, worry or being upset. Of the 714 patients studied, $40.2 \%$ reported being lonely and of those $63.8 \%$ had night-eating symptoms $\left(\chi^{2}=17.66, p<0.001\right)$. When compared to patients without night-eating symptoms, those with night-time eating symptoms were significantly more likely to be obese [OR=2.6 (95\% CI 1.5-4.5)] [49].

Another study looked at centenarians and found that those who were obese reported very good self-rated health (SRH) more often than those with normal body mass index (BMI) [OR=5.35 (95\% CI 1.67-17.18)] [47]. However, when comparing groups who rated their health as very good or good to those rating their health as poor, loneliness was significantly related to worse SRH [OR=2.89 (95\% CI 1.635.13)] \& [OR=2.23 (95\% CI 1.24-4.010] respectively. A longitudinal study followed patients from age 70 to age 77 and found that at both ages, chronic back pain was more frequent among those reporting loneliness $(p<0.001)$ and chronic back pain was associated with obesity at both ages $(\mathrm{p}=0.06$ and $\mathrm{p}=0.59$, respectively) [45]. This study also reported loneliness to be an independent risk factor at age 70 for chronic back pain [OR=4.60, (95\% CI 1.19-17.71)].

Finally, one recent study of obese female college students reported that loneliness is a significant negative emotional experience. Loneliness was reported as being inversely related to, to self-esteem $(r=-0.688, \mathrm{p}<.001)$ and a positively related to higher perceived stress levels $(r=0.560, p<.001)$ [50]. The results of this study make it logical to contemplate that the experience of loneliness may be eliciting a physiological stress response that contributes to obesity.

\section{LONELINESS AND DIABETES}

Few studies could be found that examined the significance of loneliness to diabetes. Of six potential articles identified, 2 were literature reviews, one was qualitative, and 3 involved the use of questionnaires [49, 5155]. Only one study was retrieved that used a valid and reliable measurement of loneliness [55]. While limited findings are present in the literature, some common themes emerged. Loneliness and outcomes of diabetes seem to have a reciprocal relationship. Complications of diabetes lead to decreased physical mobility which decreases an individual's ability to seek social interaction and causes strain on marital, family, and friend relationships, thus leading to loneliness [49, 51, 53-55]. Conversely, the stress induced systemic inflamm1ation observed in persons with loneliness is a known risk factor for poor health outcomes associated with diabetes $[51,52,55]$.

\section{LONELINESS AND PULMONARY PROBLEMS}

Seven articles were retrieved from the results of the EBSCOHost search of Loneliness and Pulmonary Disease and an additional 2 were reviewed from a search of Loneliness and Chronic Obstructive Pulmonary Disease. Of these articles, 2 duplicates were eliminated, one was eliminated because it was not in English even though English language was one of the selection criterion. Two articles were eliminated because they were opinion/commentary pieces and one article was eliminated because it was qualitative study.

A study of 30 people with COPD and their spouses described the prevalence of loneliness, depression, social support, and satisfaction with social support [56]. This study 
used a convenience sample and measured loneliness using the UCLA loneliness [56]. Sixty-three percent of those with COPD and, similarly, $67 \%$ of spouses reported moderate to moderately high levels of loneliness. A larger percentage of those with COPD reported high levels of depression than did their spouses. Social support and satisfaction with the social support was found to have an association with loneliness among those with COPD. Those with higher levels of social support satisfaction had lesser feelings of loneliness and depression. Among spouses it was unexpected that social support was not found to have a statistically significant correlation with loneliness.

A similar study was conducted on 30 patients with COPD and their spouses in an outpatient clinic of a university hospital in Turkey [57]. They were asked to complete the UCLA Loneliness Scale, the Back Depression Inventory and the Perceived Social Support Scales. Both patients and their spouses reported similar levels of loneliness ranging from moderately high to low levels. Nearly $97 \%$ of patients and 93\% of spouses reported levels of loneliness as moderate to moderately high. A greater number of patients $(n=18)$ with COPD rated their level of loneliness as moderately high then did the spouses $(n=11)$ and conversely less patients rated their loneliness as moderate or low $(n=12)$ than did spouses $(\mathrm{n}=19)$. Similar to the findings reported by Keele-Card et al., (1993), a significant positive association was reported between loneliness and depression in both groups. In the patients with COPD, a small inverse association existed between loneliness and perceived social support from family, and a strong negative association existed between perceived social supports from friends. In the spouses, a moderate inverse association was reported between loneliness and social support from both family and friends. The mean loneliness scores for both groups in this study were higher than those reported by Keele-Card et al.

A third study comparing the differences between clients of a Veterans Affairs Home-Based Primary Care and State Medicaid Waiver Program in Michigan found higher degrees of loneliness among the Veterans than the Medicaid clients [58]. Eighty-nine participants from the Veterans program and a weighted sample of 9,324 Medicaid participants with coronary artery disease, chronic obstructive pulmonary disease and cancer were compared using the interRAI home care (interRAI HC) assessment instrument. The interRAI HC measures disease presence and symptoms, physical and cognitive function, social support and services used. Although this study sample was not exclusive for COPD, it is worth noting that while both groups reported loneliness, Veterans reported a statistically significant higher incidence of loneliness than did Medicaid recipients. Differences in the two groups was a limitation due to the Veterans having higher functioning than the Medicaid recipients which may in part be due to the higher rates of dementia among those in the Medicaid program.

\section{DISCUSSION}

Though the majority (15 studies) of the research on loneliness and the chronic conditions included in this synthesis was conducted in the United States, the remaining studies were conducted in various locations, emphasizing that loneliness is being studied worldwide. Four studies were conducted in the Netherlands, four in Sweden, three studies in the United Kingdom, two in Israel, and one each in Turkey, Malaysia, Ireland, Canada, Greece, Finland, and South America. The widespread study locations is a true indicator that loneliness is currently viewed as a global health phenomenon that negatively impacts the outcomes of multiple chronic conditions.

The included studies varied in quantitative design and methodologies with twenty-one studies using cross-sectional methods and nine employing longitudinal study designs. Four studies reported results based on descriptive correlational design, two were experimental, two were observational, two were retrospective, one used mixed methods, one included a pre- and post-test design and one was a prospective cohort design. Sample sizes ranged from a low of 22 to 3,107 and the age range of the subjects was 17 to 109 years old. Several studies reported analyses of data from larger national studies. The UCLA Loneliness Scale and Beck Depression Inventory were the most common instruments used to assess loneliness. In addition, the de Jong-Gierveld loneliness scale, Philadelphia Geriatric Morale Scale, and single item questions were the basis for assessing loneliness in the remaining studies.

Loneliness had some reported association with all of the chronic conditions. Among older adults, loneliness was related to poorer clinical outcomes in those with heart disease and Tomaka (2006) suggested that improved belongingness support could result in improved health outcomes. Similarly, high levels of loneliness were reported among both persons with COPD and their spouses and among this population, higher levels of social support were also related to lower levels of loneliness [56, 57]. This is similar to the findings by Bergdahl et al. (2005) who found that persons living at home after a stroke, but were not alone had higher morale and thus were less lonely than those in similar home environments who have not had a stroke [37].

Additionally, physiologic measures deviating from normal for hypertension and cardiovascular reactivity were more commonly found among those who reported higher rates of loneliness [17-21, 59]. Lab value differences among persons who reported being lonely indicate that the autonomic response to chronic conditions may have a negative effect on health outcomes [20]. It is important to note that studies of adults and older adults who are healthy without chronic conditions have highlighted the presence of a physiological stress response in relation to cardiovascular disease [60-62]. These studies were not included in this review because the samples used were described as healthy individuals without heart disease. Likewise, stress induced inflammation that occurs in persons who are lonely is a risk factor for poor diabetes outcomes [52, 55, 63]. Some studies suggested that loneliness might actually be a predecessor to chronic conditions. Loneliness as a predominant characteristic in many studies showed an increase in exacerbation of symptoms and disease progression [24, 26].

Loneliness was also found to be associated with hospital readmissions, longer lengths of stay and the over utilization of healthcare resources for cardiovascular disease [25]. Heart failure and stroke were both found to be linked to higher 
depression levels after disease onset [27, 31]. Placement in a nursing home or rehabilitation facility after a stroke increases the prevalence of loneliness and depression [33, 41]. Lower socioeconomic status and living in a rural area were associated with a higher incidence of loneliness. Socioeconomic status and loneliness after stroke were found to be related to race [14].

Studies of obesity and loneliness revealed a number of factors associated with the simultaneous presence of these conditions including; poor sleep, depression, low physical activity, higher rates of night time eating and increased chronic back pain [45, 46, 49]. Weight-related stigma resulting in less success in the workplace, including not being hired or promoted and being wrongfully dismissed, and was associated with increased loneliness [44]. Loneliness was significantly associated with increasing likelihood of meeting metabolic syndrome criteria and central obesity [55]. This study was one of the few that included a measure of loneliness in people with diabetes and did find that higher rates of loneliness had a detrimental effect on diabetes outcomes.

Effective interventions for loneliness are limited and thus, are needed. One meta-analysis of interventions, that included studies across populations and age groups, reported that the most effective interventions may be those that address the ineffective or maladaptive thinking processes that occur when one is experiencing loneliness [64]. Currently, there are three interventions that have true potential for diminishing loneliness in older adults. One feasibility study focused on reducing loneliness in a sample of stroke survivors and evaluated the efficacy of an intervention that was based on videoconferencing and telehealth programs [42]. After completing the intervention, study participants reported less loneliness. More recently, investigators reported that a mindfulness-based stress reduction program resulted in lower loneliness scores and less pro-inflammatory gene expression when tested in a sample of older adults who were healthy without common chronic conditions [65]. This type of intervention has the potential to also demonstrate effectiveness in populations who suffer common chronic conditions. Most recently, the results of the first randomized trial of LISTEN (Loneliness Intervention based on Story Theory to Enhance Nursingsensitive outcomes) were reported to diminish loneliness and improve blood pressure [66, 67]. LISTEN was developed by integrating the core concepts of story theory [68] with the basic principles of cognitive restructuring and was effective for diminishing loneliness and systolic blood pressure when delivered in small groups of rural, underserved, chronically ill older adults [69]. Designing effective interventions for loneliness requires in-depth understanding of loneliness as a psychological construct that may be experienced in ways unique to an individual based on life experiences, life expectations, and cultural experience. Both LISTEN and the mindfulness intervention developed and studied by Creswell (2012) have real potential for success at diminishing loneliness in older adults and other populations and age groups.

\section{CONCLUSION}

Loneliness is known to be associated with or influence the outcomes of multiple chronic conditions including heart disease, hypertension, stroke, obesity, diabetes and pulmonary disease. Although some studies have explored the physiological mechanisms that may be triggered by loneliness, better defining these physiological links to the progression of chronic conditions would contribute to the refinement of knowledge about loneliness and health. Intervention trials aimed to diminish loneliness in persons with multiple chronic conditions have been limited, and thus, are needed. In addition, there may be opportunities for public health awareness campaigns regarding the significance of loneliness as a negative health state that may influence chronic conditions, functional ability [70, 71], and even mortality $[6,7]$. Several recent with successful programs has the potential to decrease morbidity and mortality and improve quality of life among those affected by these chronic conditions. The health care system would benefit from developing successful programs to eliminate or reduce loneliness that could potentially delay or prevent the onset of chronic disease and lessen complications and improve outcomes of those already afflicted by these chronic conditions.

\section{CONFLICT OF INTEREST}

The authors confirm that this article content has no conflicts of interest.

\section{ACKNOWLEDGEMENTS}

Declared none.

\section{REFERENCES}

[1] Theeke LA. Sociodemographic and health-related risks for loneliness and outcome differences by loneliness status in a sample of u.S. Older adults. Res Gerontol Nurs 2010; 3: 113-25.

[2] Steed L, Boldy D, Grenade L, Iredell H. The demographics of loneliness among older people in perth, western australia. Australas J Ageing 2007; 26: 81-6.

[3] Portnoy E. Loneliness - a sourcebook of current theory, research and therapy - peplau, la, perlman, d. Gerontologist 1983; 23: 32930 .

[4] Cacioppo JT, Hawkley LC, Thisted RA. Perceived social isolation makes me sad: 5-year cross-lagged analyses of loneliness and depressive symptomatology in the chicago health, aging, and social relations study. Psychol Aging 2010; 25: 453-63.

[5] Bekhet AK, Zauszniewski JA. Mental health of elders in retirement communities: Is loneliness a key factor? Arch Psychiatr Nurs 2012; 26: 214-24.

[6] Luo Y, Hawkley LC, Waite LJ, Cacioppo JT. Loneliness, health, and mortality in old age: a national longitudinal study. Soc Sci Med 2012; 74: 907-14.

[7] Perissinotto CM, Stijacic Cenzer I, Covinsky KE. Loneliness in older persons: A predictor of functional decline and deathloneliness in older persons. Arch Intern Med 2012; 172: 1078-84.

[8] Hawkley LC, Thisted RA, Masi CM, Cacioppo JT. Loneliness predicts increased blood pressure: 5-year cross-lagged analyses in middle-aged and older adults. Psychol Aging 2010; 25: 132-41.

[9] Hackett RA, Hamer M, Endrighi R, Brydon L, Steptoe A. Loneliness and stress-related inflammatory and neuroendocrine responses in older men and women. Psychoneuroendocrinology 2012; 37: 1801-9.

[10] Moher D, Liberati A, Tetzlaff J, Altman DG. Preferred reporting items for systematic reviews and meta-analyses: The prisma statement. Ann Intern Med 2009; 151: 264-9. 
[11] Lynch JJ. The broken heart: The medical consequences of loneliness. New York, NY: Basic Books, Inc 1977.

[12] Nausheen B, Gidron Y, Gregg A, Tissarchondou HS, Peveler R. Loneliness, social support and cardiovascular reactivity to laboratory stress. Stress 2007; 10: 37-44.

[13] Hawkley LC, Masi CM, Berry JD, Cacioppo JT. Loneliness is a unique predictor of age-related differences in systolic blood pressure. Psychol Aging 2006; 21: 152-64.

[14] Tomaka J, Thompson S, Palacios R. The relation of social isolation, loneliness, and social support to disease outcomes among the elderly. J Aging Health 2006; 18: 359-84.

[15] Patterson AC, Veenstra G. Loneliness and risk of mortality: a longitudinal investigation in alameda county, california. Soc Sci Med 2010; 71: 181-6.

[16] Theeke LA, Mallow J. Original research: Loneliness and quality of life in chronically ill rural older adults. AJN 2013: 113: 28-37.

[17] Cacioppo JT, Burleson MH, Poehlmann KM, et al. Autonomic and neuroendocrine responses to mild psychological stressors: Effects of chronic stress on older women. Ann Behav Med 2000; 22: 1408.

[18] Cacioppo JT, Hawkley LC, Crawford LE, et al.. Loneliness and health: potential mechanisms. Psychosom Med 2002; 64: 407-17

[19] O'Donovan A, Hughes B. Social support and loneliness in college students: effects on pulse pressure reactivity to acute stress. Int J Adolesc Med Health 2007; 19: 523-8.

[20] Norman GJ, Cacioppo JT, Morris JS, Malarkey WB, Berntson GG, Devries AC. Oxytocin increases autonomic cardiac control: moderation by loneliness. Biol Psychol 2011; 86: 174-80.

[21] Momtaz YA, Hamid TA, Yusoff S, et al.. Loneliness as a risk factor for hypertension in later life. J Aging Health 2012; 24: 696710 .

[22] Osterberg AS, Baigi A, Bering C, Fridlund B. Knowledge of heart disease risk in patients declining rehabilitation. Br J Nurs 2010; 19: 288-93.

[23] Thurston RC, Kubzansky LD. Women, loneliness, and incident coronary heart disease. Psychosom Med 2009; 71: 836-42.

[24] Tobo-Medina N, Canaval-Erazo GE. Las emociones y el estrés en personas con enfermedad coronaria. Aquichan 2010; p. 10.

[25] Lofvenmark C, Mattiasson AC, Billing E, Edner M. Perceived loneliness and social support in patients with chronic heart failure. Eur J Cardiovasc Nurs 2009; 8: 251-8.

[26] Seo Y, Yates B, Dizona P, Laframboise L, Norman J. Predictors of cognitive/affective and somatic depression in heart failure patients. Clin Nurs Res 2014; 23: 259-80.

[27] Brouwers C, Kupper N, Pelle AJ, Szabo BM, Westerhuis BL, Denollet J. Depressive symptoms in outpatients with heart failure: Importance of inflammatory biomarkers, disease severity and personality. Psychol Health 2014; 29: 564-82.

[28] Boden-Albala B, Litwak E, Elkind M, Rundek T, Sacco R. Social isolation and outcomes post stroke. Neurology 2005; 64: 1888-92.

[29] Cox AM, McKevitt C, Rudd AG, Wolfe CD. Socioeconomic status and stroke. Lancet Neurol 2006; 5: 181-8.

[30] Cacioppo JT, Hughes ME, Waite LJ, Hawkley LC, Thisted RA. Loneliness as a specific risk factor for depressive symptoms: Cross-sectional and longitudinal analyses. Psychol Aging 2006; 21 : 140-51.

[31] Ebrahim S, Barer D, Nouri F. Affective illness after stroke. Br J Psychiatry 1987; 151: 52- 6 .

[32] Narushima K, Kosier JT, Robinson RG. Preventing poststroke depression: A 12-week double-blind randomized treatment trial and 21-month follow-up. J Nerv Ment Dis 2002; 190: 296-303.

[33] Theeke L, Horstman P, Mallow J, et al.. Quality of life and loneliness in stroke survivors living in appalachia. J Neurosci Nurs 2014; 46: E3-E15 10.1097/JNN.0000000000000097.

[34] Hilari K, Northcott S, Roy P, et al. Psychological distress after stroke and aphasia: the first six months. Clin Rehabil 2010; 24: 181-90.

[35] Taylor JS. Loneliness as a way of life. Choice 2009; 46: 1586.

[36] Tilvis RS, Laitala V, Routasalo PE, Pitkala KH. Suffering from loneliness indicates significant mortality risk of older people. J Aging Res 2011; 2011: 534781

[37] Bergdahl E, Gustavsson J, Kallin K, et al. Depression among the oldest old: The umeå 85+ study. Int Psychogeriatr 2005; 17: 55775 .

[38] Health NCCfM. In Case identification included and excluded studies. British Psychological Society, 2010.
[39] Kramer SE, Kapteyn TS, Kuik DJ, Deeg DJ. The association of hearing impairment and chronic diseases with psychosocial health status in older age. J Aging Health 2002; 14: 122-37.

[40] Wyller TB, Holmen J, Laake P, Laake K. Correlates of subjective well-being in stroke patients. Stroke 1998; 29: 363-7.

[41] Jongenelis K, Pot A, Eisses A, Beekman A, Kluiter H, Ribbe M. Prevalence and risk indicators of depression in elderly nursing home patients: The aged study. J Affect Disord 2004; 83: 135-42.

[42] Taylor DM, Cameron JI, Walsh L, et al. Exploring the feasibility of videoconference delivery of a self-management program to rural participants with stroke. Telemed J E-Health 2009; 15: 646-54.

[43] Penninx BW, Leveille S, Ferrucci L, Van Eijk J, Guralnik JM. Exploring the effect of depression on physical disability: Longitudinal evidence from the established populations for epidemiologic studies of the elderly. Am J Public Health 1999; 89: 1346-52.

[44] Chen E, Fettich K, McCloskey M. Correlates of suicidal ideation and/or behavior in bariatric-surgery-seeking individuals with severe obesity. Crisis 2012; 33: 137.

[45] Jacobs JM, Cohen A, Hammerman-Rozenberg R, Stessman J. Global sleep satisfaction of older people: The jerusalem cohort study. J Am Geriatr Soc 2006; 54: 325-9.

[46] Jacobs JM, Hammerman-Rozenberg R, Cohen A, Stessman J. Chronic back pain among the elderly: Prevalence, associations, and predictors. Spine 2006; 31: E203-E207.

[47] Tigani X, Artemiadis AK, Alexopoulos EC, Chrousos GP, Darviri C. Self-rated health in centenarians: A nation-wide cross-sectional greek study. Arch Gerontol Geriatr 2012; 54: e342-e348.

[48] Whisman MA. Loneliness and the metabolic syndrome in a population-based sample of middle-aged and older adults. Health Psychol 2010; 29: 4.

[49] Morse SA, Ciechanowski PS, Katon WJ, Hirsch IB. Isn't this just bedtime snacking? The potential adverse effects of night-eating symptoms on treatment adherence and outcomes in patients with diabetes. Diab Care 2006; 29: 1800-4.

[50] Smith MJ, Theeke L, Culp S, Clark K, Pinto S. Psychosocial variables and self-rated health in young adult obese women. Appl Nurs Res 2014; 27: 67-71.

[51] Jaremka LM, Lindgren ME, Kiecolt-Glaser J. Synergistic relationships among stress, depression, and troubled relationships: Insights from psychoneuroimmunology. Depress Anxiety (10914269) 2013; 30: 288-96.

[52] Nefs G, Pouwer F, Pop V, Denollet J. Type d (distressed) personality in primary care patients with type 2 diabetes: Validation and clinical correlates of the ds14 assessment. J Psychosom Res 2012; 72: 251-7.

[53] Ribu L, Wahl A. Living with diabetic foot ulcers: a life of fear, restrictions, and pain. Ostomy/wound Manage 2004; 50: 57-67.

[54] Vitaliano PP, Murphy M, Young HM, Echeverria D, Borson S. Does caring for a spouse with dementia promote cognitive decline? A hypothesis and proposed mechanisms. J Am Geriatr Soc 2011; 59: 900-8

[55] Whisman MA. Loneliness and the metabolic syndrome in a population-based sample of middle-aged and older adults. Health Psychol 2010; 29: 550-4.

[56] Keele-Card G, Foxall MJ, Barron CR. Loneliness, depression, and social support of patients with copd and their spouses. Public Health Nurs 1993; 10: 245-51.

[57] Kara M, Mirici A. Loneliness, depression, and social support of turkish patients with chronic obstructive pulmonary disease and their spouses. J Nurs Scholarsh 2004; 36: 331-6.

[58] Wharton TC, Nnodim J, Hogikyan R, et al. Assessing health status differences between veterans affairs home-based primary care and state medicaid waiver program clients. J Am Med Dir Assoc 2013: 14: 260-4.

[59] Nausheen B, Carr NJ, Peveler RC, et al. Relationship between loneliness and proangiogenic cytokines in newly diagnosed tumors of colon and rectum. Psychosom Med 2010; 72: 912-6.

[60] Hackett RA, Hamer M, Endrighi R, Brydon L, Steptoe A. Loneliness and stress-related inflammatory and neuroendocrine responses in older men and women. Psychoneuroendocrinology 2012.

[61] Steptoe A, Kivimäki M. Stress and cardiovascular disease. Nat Rev Cardiol 2012; 9: 360-70.

[62] Steptoe A, Owen N, Kunz-Ebrecht, SR, Brydon L. Loneliness and neuroendocrine, cardiovascular, and inflammatory stress responses 
in middle-aged men and women. Psychoneuroendocri-nology 2004; 29: 593-611.

[63] Jaremka LM, Fagundes CP, Glaser R, Bennett JM, Malarkey WB, Kiecolt-Glaser JK. Loneliness predicts pain, depression, and fatigue: Understanding the role of immune dysregulation. Psychoneuroendocrinology 2013; 38: 1310-17.

[64] Masi CM, Chen HY, Hawkley LC, Cacioppo JT. A meta-analysis of interventions to reduce loneliness. Pers Soc Psychol Rev 2011; 15: 219-66.

[65] Creswell JD, Irwin MR, Burklund LJ, et al. Mindfulness-based stress reduction training reduces loneliness and pro-inflammatory gene expression in older adults: a small randomized controlled trial. Brain Behav Immun 2012; 26: 1095-101.

[66] Theeke LA, Mallow JA, Barnes E, Barr TL, Theeke E. In The results of the first randomized trial of listen, 2014 State of the Science Congress on Nursing Research: Optimizing Health by Addressing Complexity, Washington, DC 2014.
[67] Theeke LA. In The development of listen (loneliness intervention using story theory to enhance nursing-sensitive outcomes): Integrating state fo the science knowledge about a health phenomenon into a cognitive behavioral intervention, 2014 State of the Science Congress on Nursing Research: Optimizing Health by Addressing Complexity, Washington, DC 2014.

[68] Liehr P, Smith M. Story theory. In: Middle range theory for nursing, $2^{\text {nd }}$ ed. New York: Springer Publishing Company 2008; pp. 205-24.

[69] Theeke LA, Mallow JA. The development of listen: a novel intervention for loneliness. Open J Nurs 2015; 5: 136.

[70] Warner DF, Kelley-Moore J. The social context of disablement among older adults: Does marital quality matter for loneliness? J Health Soc Behav 2012; 53: 50-66.

[71] Theeke LA, Goins RT, Moore J, Campbell H. Loneliness, depression, social support, and quality of life in older chronically ill appalachians. J Psychol 2012; 146: 155-71.

(C) Petitte et al..; Licensee Bentham Open.

This is an open access article licensed under the terms of the Creative Commons Attribution Non-Commercial License (http://creativecommons.org/licenses/by-nc/3.0/) which permits unrestricted, non-commercial use, distribution and reproduction in any medium, provided the work is properly cited. 